\title{
Proactive Spectrum Sharing for SWIPT in MIMO Cognitive Radio Systems Using Antenna Switching Technique
}

\author{
Fatma Benkhelifa*, Student Member, IEEE, \\ Kamel Tourki**, Senior Member, IEEE, and Mohamed-Slim Alouini*, Fellow, IEEE
}

\begin{abstract}
In this paper, we consider the simultaneous wireless information and power transfer (SWIPT) for the spectrum sharing (SS) in a multiple-input multiple-output (MIMO) cognitive radio (CR) network. The secondary transmitter (ST) selects only one antenna which maximizes the received signal-to-noise ratio $(\mathrm{SNR})$ at the secondary receiver $(\mathrm{SR})$ and minimizes the interference induced at the primary receiver (PR). Moreover, PR is an energy harvesting (EH) node using the antenna switching (AS) which assigns a subset of its antennas to harvest the energy and assigns the rest to decode its information data. The objective of this work is to show that the SS is advantageous for both SR and PR sides and leads to a win-win situation. To illustrate the incentive of the SS in CR network, we evaluate the energy and data performance metrics in terms of the average harvested energy, the power outage, and the mutual outage probability (MOP) which declares a data outage event if the PR or SR is in an outage. We present some special cases and asymptotic results of the derived analytic results. Through the simulation results, we show the impact of various simulation parameters and the benefits due to the presence of ST.
\end{abstract}

Index Terms-Cognitive radio (CR), spectrum sharing (SS), energy harvesting (EH), antenna switching (AS), transmit antenna selection, average harvested energy, power outage (PO), primary/secondary data outage probability, mutual outage probability (MOP).

\section{INTRODUCTION}

Simultaneous wireless information and power transfer (SWIPT) is a promising technique to ensure the selfsustainability and continuous operation of wireless communication systems. SWIPT technique allows the simultaneous use of the radio frequency (RF) signals for wireless power transfer (WPT) and wireless information transfer (WIT). The pioneering papers [1] and [2] have studied the SWIPT in singleinput single-output (SISO) through flat fading and frequency selective channels, respectively. The authors have investigated the optimal tradeoff between the information rate and the

* F. Benkhelifa and M.-S. Alouini are with the Division of Computer, Electrical, and Mathematical Sciences \& Engineering (CEMSE), King Abdullah University of Science and Technology (KAUST), Thuwal, Makkah Province, Saudi Arabia. [e-mail: \{fatma.benkhelifa, slim.alouini\}@kaust.edu.sa]. ** K. Tourki is with the Mathematical and Algorithmic Sciences Lab, France Research Center, Huawei Technologies Co. Ltd, France. [e-mail: \{kamel.tourki\}@huawei.com].

This paper was funded by a grant from Sensor Research Initiative funded by the Office of Sponsored Research at King Abdullah University of Science and Technology (KAUST), Thuwal, Saudi Arabia.

This paper was accepted in part at IEEE International Conference on Communications Workshops (ICC'2016), Kuala Lumpur, Malaysia. energy transfer for the co-located information decoding (ID) and energy harvesting $(\mathrm{EH})$ receivers. The work done in [1] and [2] was extended in [3] where two practical SWIPT schemes were presented for the multiple-input multiple-output (MIMO) communication systems. The practical schemes are the power splitting (PS) and the time switching (TS) that separate the ID and EH transfer over the power domain and the time domain, respectively. In [4], a MIMO decode-andforward (DF) relay system was considered where the source and the destination have single antennas, and the relay is an EH multi-antenna node. The authors investigated a low complexity SWIPT technique which is the antenna switching (AS) policy where the strongest antennas are exploited for ID while the others are used for EH (or vice versa). In [5], the sum rate maximization problem was considered for the two-user multiple-input single-output (MISO) interference channel. The authors have shown that even though the interference limits the achievable rate, it helps to gain more energy at the EH receivers. In [6], the data outage probability and ergodic capacity were investigated in an amplify-andforward (AF) relay system with a multi-antenna $\mathrm{EH}$ relay and with/without the presence of an interferer. Different linear processing schemes were considered, and the diversity gain and the optimal power splitting ratio were characterized. In [7], the application of smart antennas technologies were investigated using the SWIPT technique with MIMO and relaying techniques and they were shown to improve the energy and spectral efficiencies of SWIPT. In [8], [9], the source/relay precoding design was investigated in MIMO relay system with an EH relay and possibly imperfect channel state information (CSI) considering DF and AF relaying protocol, respectively.

On the other hand, cognitive radio (CR) networks is a promising technique introduced by Mitola to solve the spectrum scarcity of the wireless communication systems [10]. Indeed, CR networks allow the unlicensed users (or the secondary users (SUs)) to use the spectrum only when the licensed users (or the primary users (PUs)) are idle. Spectrum sharing (SS) in CR networks allow the SUs to share the spectrum bands as long as the interference of the latter does not harm the PUs [11]. Many works have studied the secondary capacity with single/multiple antennas at the primary and secondary networks [12]-[16]. In [12], a ratio selection scheme using a mean value (MV)-based power allocation strategy for MIMO CR networks was studied with average/peak interference power and peak transmit power 
constraints. When the secondary receiver (SR) has multiple antennas, the maximum ratio combining (MRC) technique was investigated for CR networks in [13], [14]. In [15], a joint transmit-receive antenna selection in MIMO CR network was proposed to maximize the $\mathrm{CR}$ data rates satisfying interference constraints at primary receivers (PRs) by using two approaches: one based on iterative approach and one normbased selection approach. Near-optimality of the norm-based approach was shown compared to the exhaustive search. In [16], a novel and optimal transmit antenna selection at the secondary transmitter (ST) was proposed to minimize the symbol error probability (SEP) of an average interferenceconstrained multiple-input single-output (MISO) secondary system in an underlay CR network.

\section{A. Related Works}

So far, the energy efficiency and the spectrum scarcity are two major issues in wireless communication systems. Subsequently, it is appealing to study the performance of the SWIPT technique in CR networks. Recently, some research interest has been carried out to study the SWIPT technique in CR networks [17]-[25]. In [17], an opportunistic spectrum access scheme was considered in CR networks where STs either harvest energy from ambient transmissions or transmit signals when primary transmitters (PTs) are far away. In [18], the rate maximization problem was considered where multiple PRs have EH capabilities by proving that the transmit covariance matrix is ranked one and by using the semidefinite programming (SDP) relaxation. Two suboptimal solutions were proposed to provide the lower and upper bounds of the optimal solution. In [19], the expressions of the throughput in delay-tolerant and delay-sensitive transmission modes were investigated in an underlay cognitive relay network with multiple PTs where the secondary nodes can harvest energy from the primary network. In [20], the expression of the secondary data outage probability under the primary data outage constraint and the peak power constraint at both ST and relay was investigated in SS in cognitive relay network with multiple PTs and EH secondary relay. In addition, the improvement regarding the secondary data outage probability was shown due to the interference from the primary network. In [21], the secondary data outage probability is investigated in an underlay DF relay cognitive network where a secondary relay harvests energy from the secondary source to transmit to the secondary destination. An interference constraint is imposed at PR, and an EH constraint is imposed at the secondary relay. The optimal $\mathrm{EH}$ duration was also investigated. In [22], the primary and secondary rates were investigated in a cognitive radio network composed of a primary transmitterreceiver pair and multiple secondary transmitter-receiver pairs where the primary user releases a proportion of its time slot to the secondary nodes to send their data and to power the EH primary transmitter. Various joint information and energy cooperative schemes were proposed using $\mathrm{EH}$ techniques and sparse-approximation principles. In [23], the secondary ergodic capacity, the average symbol error rate (SER), the average throughput, and the average $\mathrm{EH}$ were investigated in an underlay cognitive network with an ST communicating with multiple EH SRs and a primary transmitter-receiver pair. A channel quality based threshold and an opportunistic scheduling were exploited where the SRs with channel conditions above a predefined threshold are scheduled for data transmission, and those below the threshold are scheduled for EH. The optimal threshold was defined in a way to satisfy a target data outage probability. In [24], the exact and asymptotic data outage probability were investigated for underlay multihop cognitive relay networks where the secondary users are powered by a dedicated power beacon (PB) and their transmit powers are subject to the harvested energy from $\mathrm{PB}$ and the interference constraint at PR. In [25], the data outage probability and the high signal-to-noise (SNR) approximations were determined in an underlay cognitive relay network with one PR, one secondary transmitter-receiver pair, and an $\mathrm{EH}$ relay. An interference constraint at $\mathrm{PR}$ and an $\mathrm{EH}$ constraint at the relay were imposed.

\section{B. Contributions}

In this paper, we propose to study the SWIPT system for the SS in CR networks where PR and ST have multiple antennas. The PR uses the AS technique which allocates a subset of the receiving antennas to harvest the required energy for its operation and the remaining receiving antennas to decode the information data. The ST selects only one antenna to transmit. The presence of ST helps to improve the $\mathrm{EH}$ requirements at PR since the interference boosts the energy gains at the $\mathrm{EH}$ receivers. Consequently, fewer antennas at PR are connected to the $\mathrm{EH}$ receivers, and more antennas at $\mathrm{PR}$ are assigned to the ID receivers to compensate the data losses due to the interference. Hence, we have a win-to-win situation at both PR and SR ends. To illustrate the incentive of SS in CR networks, we study the energy and data performance of our system in terms of the average harvested energy $\bar{Q}$., the power outage $\mathcal{P O}$, and the mutual outage probability (MOP) defined in [26]. The main contributions of this work are listed as follows:

- An antenna selection scheme at ST is proposed in a way to maximize the channel gain between ST and SR and minimize the global interference induced by ST at PR. Closed-form expressions of the probability distribution function (PDF) and cumulative distribution function (CDF) of the channel gain between the selected antenna at ST and SR were derived in Lemma 1.

- Closed form expressions of the CDF and PDF of the harvested energy at PR were derived for a given number of selected antennas $K=0, \ldots, N_{p}-1$ to decode the information data in Lemma 2.

- An EH model at the SWIPT-constrained multi-antenna PR is proposed based on the AS scheme where $K$ should respond to some energy requirements $E_{t}$ at PR. Based on the proposed EH model, the probability mass function (PMF) of the number of selected antennas $K$ at PR was derived in Theorem 1. The closed form expressions of the average harvested energy and the power outage at PR were also derived.

- The primary and secondary data outage probabilities were derived in Theorem 2 and Theorem 3, respectively. Using 


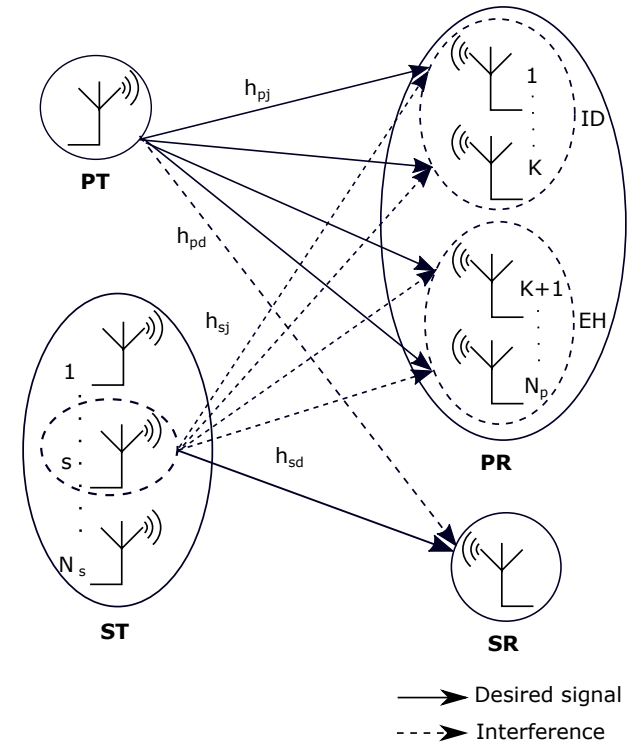

Figure 1. System Model

the results in Theorem 2 and Theorem 3, the closed form expression of the MOP, defined in [26] which declares a data outage when either the PR or SR is in an outage, is derived.

- Relevant special cases were discussed: single antenna at ST, maximum channel gain antenna selection at ST, absence of ST, and TS scheme at PR.

- Asymptotic results of all the analytic results were derived in the low/high transmit power regime at PT and ST and in the low/high energy threshold regime.

- Through the simulation results, we have shown the benefits due to the presence of ST in terms of data and energy metrics. Also, the AS scheme mostly performs better that the TS scheme in terms of the data metric $\mathcal{M O P}$ and the energy metrics $\mathcal{P} O$, and $\bar{Q}$. Moreover, we have shown the impact of various simulation parameters (number of antennas, transmit power, interference channel variance, transmission rate, energy threshold, etc.) on all the analytic results of the data and energy performance metrics.

\section{System Model}

As shown in Fig. 1, we consider a SWIPT-enabled cognitive network consisting of a primary transmitter-receiver pair and a secondary transmitter-receiver pair. While all the nodes are battery powered, the PR is an EH node. The PT and SR are equipped with a single antenna, while the PR and ST have $N_{p}$ and $N_{s}$ antennas, respectively. The channel between PT and the $j$ 'th antenna of PR, the channel between PT and SR, the channel between the $l^{\prime}$ th antenna of ST and the $j^{\prime}$ th antenna of PR, and the channel between the $l^{\prime}$ th antenna of ST and SR are denoted by $h_{p j}, h_{p d}, h_{l j}$, and $h_{l d}$, respectively, $\forall j=$ $1, \ldots, N_{p}$ and $\forall l=1, \ldots, N_{s}$. All the channels $h_{p j}, h_{p d}, h_{l j}$ and $h_{l d}$ are modeled as flat fading with Rayleigh distribution, with variances $\lambda_{p p}, \lambda_{p d}, \lambda_{s p}$, and $\lambda_{s d}$, respectively. We assume that CSI is perfectly known at all the nodes. The transmit powers at PT and ST are denoted by $P_{p}$ and $P_{s}$, respectively, and the variances of the additive white Gaussian noise (AWGN) at PR and SR are denoted by $\sigma_{p}^{2}$ and $\sigma_{s}^{2}$, respectively.

In the sequel, we propose to use a broadcasting scheme at ST to select only one transmit antenna in a way to maximize the channel gain between ST and SR and minimize the effect of interference induced at PR. Moreover, we use the AS technique at the self-powered PR by allocating a subset of its receiving antennas to harvest the energy and the rest to decode the information data.

The self-powered PR uses the AS technique to harvest the energy. Indeed, there are several types of practical $\mathrm{EH}$ schemes [3], [4] that separate the ID and EH processes over separate resources such as the time slot, the frequency band, the power fraction. Due to its low complexity and ease of deployment, we opt to use the AS technique that separates the ID and EH processes over the spatial diversity at the multiantenna PR. Moreover, the interference due to ST presence is usually unwanted due to its drawbacks on the information data processing. However, because of the SWIPT constraint at PR, the interference can be highly appreciated to allow the PR to respond to its energy requirement at PR with less number of $\mathrm{EH}$ antennas and hence it allows to allocate more number of ID antennas to combat the negative effect of the interference on the primary data throughput. At this point, we can see that the interference is desired at the EH receiving antennas while it is undesired at the ID antennas.

\section{A. Transmit Antenna Selection at ST}

Since the ST is equipped with multiple antennas, we propose to use a broadcasting scheme at ST that selects only one antenna out of $N_{s}$ antennas in a way to maximize the channel gain between ST and SR, while minimizing the effect of interference induced at PR. Note that using all antennas at ST while achieving both goals is quiet challenging and out of the scope of this work. Moreover, this broadcasting is beneficial to PR which will transform a part of this interference into useful energy (interference recycling). Using beamforming at ST is not interesting in our case since it will decrease the amount of energy that PR can scavenge. Now, let us explain how to perform the transmit antenna selection at ST.

The ST, equipped with $N_{s} \geq 1$ antennas, selects only one transmit antenna $s$ to transmit to SR under the condition that

$$
s=\underset{1 \leq l \leq N_{s}}{\arg \max } \frac{\left|h_{l d}\right|^{2}}{\sum_{j=1}^{N_{p}}\left|h_{l j}\right|^{2}} .
$$

This antenna selection scheme aims to maximize the channel gain between ST and SR while minimizing the interference induced at PR. It is worth noting that PR cannot expect in advance which antenna subset will be selected for ID. For this reason, ST selects the transmitting antenna while considering that all PR antennas will be selected for ID. This selection scheme was inspired by the well known selection scheme in the non-EH MIMO cognitive radio networks, which is the ratio selection scheme [12], [27]. In that case, the signal-tointerference-plus-noise ratio (SINR) at SR is written as

$$
\gamma_{S}=\frac{\frac{P_{s}}{\sigma_{s}^{2}}\left|h_{s d}\right|^{2}}{\frac{P_{p}}{\sigma_{s}^{2}}\left|h_{p d}\right|^{2}+1}=\frac{\gamma_{s d}}{\gamma_{p d}+1},
$$


where $\gamma_{s d}=\overline{\gamma_{s_{1}}}\left|h_{s d}\right|^{2}, \gamma_{p d}=\overline{\gamma_{s_{2}}}\left|h_{p d}\right|^{2}, \overline{\gamma_{s_{1}}}=\frac{P_{s}}{\sigma_{s}^{2}}$, and $\overline{\gamma_{s_{2}}}=\frac{P_{p}}{\sigma_{s}^{2}}$. Let us denote by $\alpha_{1}=\frac{P_{s}}{\sigma_{s}^{2}} \lambda_{s d}$, and $\alpha_{2}=\frac{P_{p}}{\sigma_{s}^{2}} \lambda_{p d}$.

Now, let us present some relevant statistics that we will help to evaluate the performance of the secondary network.

Lemma 1: The PDF and CDF of $\gamma_{s d}$ are not trivially obtained and their expressions are given by

$$
\begin{aligned}
f_{\gamma_{s d}}(x) & =\frac{N_{s}}{\alpha_{1}} e^{-\frac{x}{\alpha_{1}}}+\frac{N_{s}}{\Gamma\left(N_{p}\right) \alpha_{1}} \sum_{j=1}^{N_{s}-1} \sum_{i=0}^{N_{p}(j+1)-1}\left(\begin{array}{c}
N_{s}-1 \\
j
\end{array}\right) \\
& \times\left(\begin{array}{c}
N_{p}(j+1)-1 \\
i
\end{array}\right)(-1)^{j+i}\left(\frac{x}{\alpha_{1}}\right)^{i} \Gamma\left(N_{p}-i, \frac{x}{\alpha_{1}}\right), \\
\mathcal{F}_{\gamma_{s d}}(y) & =N_{s}\left(1-e^{-\frac{y}{\alpha_{1}}}\right)+\frac{N_{s}}{\Gamma\left(N_{p}\right) \alpha_{1}} \sum_{j=1}^{N_{s}-1} \sum_{i=0}^{N_{p}(j+1)-1}\left(\begin{array}{c}
N_{s}-1 \\
j
\end{array}\right) \\
& \times\left(\begin{array}{c}
N_{p}(j+1)-1 \\
i
\end{array}\right) \frac{(-1)^{j+i}}{i+1}\left[\gamma\left(N_{p}+1, \frac{y}{\alpha_{1}}\right)\right. \\
& \left.+\alpha_{1}\left(\frac{y}{\alpha_{1}}\right)^{i+1} \Gamma\left(N_{p}-i, \frac{y}{\alpha_{1}}\right)\right],
\end{aligned}
$$

for $x \geq 0$, and $y \geq 0$, where $\Gamma(\cdot)$ is the Gamma function, and $\Gamma(\cdot, \cdot)$ and $\gamma(\cdot, \cdot)$ are the upper and lower incomplete Gamma function [28], respectively.

Proof: The proof is derived in Appendix A.

\section{B. Antenna Switching (AS) at PR}

The self-powered PR uses the AS technique to harvest the energy. Indeed, there are several types of practical EH schemes [3], [4] that allocate separate resources to perform the ID and EH processes, such as the time slot, the frequency band, the power fraction. Here, we opt to use the AS technique that separates the ID and EH processes over the spatial diversity at the multi-antenna PR [4]. First, we choose the AS scheme due to its low complexity and ease of deployment, compared to the other EH schemes. Moreover, the separation between the ID and EH modes over space in the AS scheme will help to make use of the interference induced by ST. At the EH receiving antennas, the interference is highly desired to improve the harvested energy at PR. However, at the ID antennas, the interference is usually undesired due to its drawbacks on the information data processing at PR. But, if the ST presence helps the PR to respond to its energy requirements with fewer number of EH antennas than the case without ST interference, this case will allow the PR to allocate more number of ID antennas to combat the negative effect of the interference on the primary data throughput, and hence, we have a win-towin situation. So, using the AS scheme, the usefulness of the interference is separated where it is appreciated at the $\mathrm{EH}$ receiving antennas while it is mitigated at the ID receiving antennas. But, if we use for example the power splitting scheme, the interference is simultaneously used for ID and $\mathrm{EH}$ and its usefulness is not highlighted.

The PR is equipped with $N_{p}$ antennas. In the AS scheme, each receiving antenna is either connected to the EH circuit or the ID circuit. Let $K$ be the number of antennas at PR connected to the ID circuits and $N_{p}-K$ be the number of antennas at PR connected to the EH circuits. The antennas are randomly ordered [4]. For a given $K \in\left\{1, \ldots, N_{p}\right\}$, the combined SINR at PR, after performing the MRC technique in the presence of co-channel interference $(\mathrm{CCI})$ as shown in [29], is given by

$$
\gamma_{I D}(K)=\frac{\frac{P_{p}}{\sigma_{p}^{2}} \sum_{j=1}^{K}\left|h_{p j}\right|^{2}}{\frac{P_{s}}{\sigma_{p}^{2}} \frac{\left|\sum_{j=1}^{K} h_{p j}^{*} h_{s j}\right|^{2}}{\sum_{j=1}^{K}\left|h_{p j}\right|^{2}}+1}=\frac{\gamma_{p p}}{\gamma_{s p}+1},
$$

where $\gamma_{p p}=\overline{\gamma_{p_{1}}} g_{p p}, g_{p p}=\sum_{j=1}^{K}\left|h_{p j}\right|^{2}, \overline{\gamma_{p_{1}}}=\frac{P_{p}}{\sigma_{p}^{2}}, \gamma_{s p}=\overline{\gamma_{p_{2}}} g_{s p}$, $g_{s p}=\frac{\left|\sum_{j=1}^{K} h_{p j}^{*} h_{s j}\right|^{2}}{\sum_{j=1}^{K}\left|h_{p j}\right|^{2}}$, and $\overline{\gamma_{p_{2}}}=\frac{P_{s}}{\sigma_{p}^{2}}$. The PDF of $\gamma_{s p}$ was shown in [29] that it is independent of $\gamma_{p p}$ and it follows the exponential distribution. Let us denote by $\beta_{1}=\frac{P_{p}}{\sigma_{p}^{2}} \lambda_{p p}$ and $\beta_{2}=\frac{P_{s}}{\sigma_{p}^{2}} \lambda_{s p}$. Moreover, the harvested energy at PR is given by

$$
Q(K)= \begin{cases}\sum_{j=K+1}^{N_{p}} E_{j}, & \text { if } 0 \leq K<N_{p}, \\ 0, & \text { if } K=N_{p},\end{cases}
$$

where $E_{j}=\zeta T\left(P_{p}\left|h_{p j}\right|^{2}+P_{s}\left|h_{s j}\right|^{2}\right)$ is the harvested energy at the j'th antenna of PR, $\zeta \in[0$,$] is the conversion efficiency,$ and $T$ is the period duration which is normalized for simplicity without any loss of generality. Let us denote by $\varepsilon_{p}=P_{p} \lambda_{p p}$, $\varepsilon_{s}=P_{s} \lambda_{s p}$, and $\varepsilon_{p s}=\varepsilon_{p}-\varepsilon_{s}$.

In what follows, we present some relevant statistics useful to approach the performance of the primary network. Let $f^{\left(N_{p}-k\right)}(\cdot)$ and $\mathcal{F}^{\left(N_{p}-k\right)}(\cdot)$ be the PDF and CDF of $Q(k)$, $\forall k=0, \ldots, N_{p}-1$. Their expressions are given in the following lemma and are depending on the value of $\varepsilon_{p s}$ either zero or not.

Lemma 2: The PDF and CDF of $Q(k)$, for $k=0, \ldots, N_{p}-1$, are given by

$$
f^{\left(N_{p}-k\right)}(z)= \begin{cases}\frac{1}{\Gamma\left(2\left(N_{p}-k\right)\right)} \frac{z^{2\left(N_{p}-k\right)-1}}{\left(\zeta \varepsilon_{s}\right)^{2\left(N_{p}-k\right)}} e^{-\frac{z}{\zeta \varepsilon_{s}}}, & \text { if } \varepsilon_{p s}=0, \\ \frac{\sqrt{\pi} z^{N_{p}-k-\frac{1}{2}} e^{-\left(\frac{1}{\varepsilon_{s}}+\frac{1}{\varepsilon_{p}}\right) \frac{z}{2 \zeta}}}{\Gamma\left(N_{p}-k\right) \zeta \sqrt{\varepsilon_{p} \varepsilon_{s}}\left(\zeta \varepsilon_{p s}\right)^{N_{p}-k-\frac{1}{2}}} I_{N_{p}-k-\frac{1}{2}}\left(\frac{\varepsilon_{p s}}{\zeta \varepsilon_{p} \varepsilon_{s}} \frac{z}{2}\right), & \text { if } \varepsilon_{p s} \neq 0,\end{cases}
$$$$
\mathcal{F}^{\left(N_{p}-k\right)}(t)= \begin{cases}\frac{\gamma\left(2\left(N_{p}-k\right), \frac{t}{\zeta \varepsilon_{s}}\right)}{\Gamma\left(2\left(N_{p}-k\right)\right)}, & \text { if } \varepsilon_{p s}=0, \\ \frac{\gamma\left(N_{p}-k, \frac{t}{\zeta \varepsilon_{p}}\right)}{\Gamma\left(N_{p}-k\right)}-\psi_{k}(t), & \text { if } \varepsilon_{p s} \neq 0,\end{cases}
$$

where $z \geq 0$, and $t \geq 0, I_{v}(\cdot)$ is the modified Bessel function of the first kind, $\psi_{k}(x)$ is defined in $(9)$, and ${ }_{1} F_{1}(a ; b ; z)$ is the Kummer confluent hypergeometric function [28].

Proof: The proof is derived in Appendix B.

From (6), we can see that $Q(K+1)<Q(K)$. Hence, $Q(K)$ is strictly decreasing with respect to $K$.

Remark 1: Note that if only one antenna is selected to harvest the energy and the remaining antennas are used to decode the information data, i.e. $k=N_{p}-1$, the PDF and CDF of $Q(k)$ simplifies to

$$
\begin{aligned}
& f^{(1)}(z)=\left\{\begin{array}{l}
\frac{z e^{-\frac{z}{\zeta s}}}{\left.\zeta \varepsilon_{s}\right)^{2}}, \text { if } \varepsilon_{p s}=0, \\
\frac{e^{-\frac{\varepsilon_{p}}{\zeta \varepsilon_{p}}}-e^{-\frac{z}{\zeta \varepsilon_{s}}}}{\zeta \varepsilon_{p s}}, \text { if } \varepsilon_{p s} \neq 0,
\end{array}\right. \\
& \mathcal{F}^{(1)}(t)= \begin{cases}1-\left(1+\frac{t}{\frac{t}{\zeta \varepsilon_{s}}}\right) e^{-\frac{t}{\zeta \varepsilon_{s}}}, & \text { if } \varepsilon_{p s}=0, \\
1-\frac{\varepsilon_{p} e^{\frac{t}{\zeta \varepsilon_{p}}}-\varepsilon_{s} e^{\frac{t}{\varepsilon_{s}}}}{\varepsilon_{p s}}, & \text { if } \varepsilon_{p s} \neq 0,\end{cases}
\end{aligned}
$$

for $z \geq 0$, and $t \geq 0$. 


$$
\psi_{k}(t)=\left(\frac{t}{\zeta \varepsilon_{p}}\right)^{N_{p}-k} e^{-\frac{t}{\zeta \varepsilon_{s}}} \sum_{j=0}^{N_{p}-k-1} \frac{\left(\frac{t}{\zeta \varepsilon_{s}}\right)^{j}{ }_{1} F_{1}\left(N_{p}-k ; N_{p}-k+j+1 ; \frac{\varepsilon_{p s}}{\zeta \varepsilon_{s} \varepsilon_{p}} t\right)}{\Gamma\left(N_{p}-k+j+1\right)}
$$

\section{EH Model AT PR}

Let us consider the EH model explaining how to choose the number of antennas $K$ out of $N_{p}$ at PR connected to the ID circuits. The selection of the number of antennas $K$ is important as it highly affects the performance of PR. If $K$ is close to zero, more energy is harvested while less data is decoded. However, if $K$ is close to $N_{p}$, more data is decoded, and less energy is harvested. How to choose $K$ in a way to satisfy the energy requirement at PR while decoding the maximum data is explained below.

\section{A. How to Choose $K$}

First, let us denote by:

- $E_{0}$ : the state of the battery at instant 0 . We assume that PR is equipped with a battery with infinite capacity.

- $E_{m}$ : the minimum required energy to be available in the battery.

- $E_{p}$ : the energy to be used by the ID and EH circuits during a period $T$. For a given $E_{0}$ and a given $K$, we assume that $E_{p}$ is known and constant over the time. Let us denote by $E_{t}=E_{p}+E_{m}-E_{0}$, the energy threshold.

- $E_{s}$ : the state of the battery at the beginning of the transmission phase.

Without loss of generality, the transmission phases last over $n T \leq t<(n+1) T$, where $n$ is an integer. For the sake of simplicity, we omit the time index. For a given initial state of battery $E_{0}$ and a given selected number of antennas $K=$ $0, \ldots, N_{p}$, the state of the battery at the end of the transmission phase is given by

$$
E_{s}(K)=E_{0}-E_{p}+Q(K),
$$

which should be greater than the minimum required harvested energy $E_{m}$, as

$$
E_{s}(K) \geq E_{m} \Leftrightarrow E_{0}+Q(K) \geq E_{p}+E_{m} .
$$

The decision on either to harvest the energy or not depends on the state of the battery if we don't harvest energy, which is equal to the energy threshold $E_{t}$. If we do not harvest energy and we are still above the threshold $E_{m}$, then there is no interest of harvesting energy. Otherwise, we need to harvest the energy. The amount to be harvested depends on $E_{t}$ and $Q(k), \forall k=$ $0, \ldots N_{p}-1$. In fact, we observe that

- If $E_{t} \leq 0$, no harvesting is needed. Hence, all antennas are used to decode the information data, i.e. $K=N_{p}$ and $Q(K)=0$.

- If $E_{t}>0, K$ is selected such that $Q(K) \geq E_{t}, 0 \leq K \leq$ $N_{p}-1$. Since $Q(k)$ is strictly decreasing with respect to $K$, the best choice of $K$ should satisfy

$$
Q(K+1)<E_{t} \leq Q(K)
$$

But, if $E_{t}$ is strictly greater than $Q(1)$, the condition in (13) cannot be satisfied and hence the choice of $K$ cannot be performed as in (14). In this case, even if all the antennas are used for $\mathrm{EH}$, they are not enough to reach the minimum required energy $E_{m}$. So, we have an energy outage. For simplicity, we take $K$ equal to zero. In the next transmission phase, $E_{0}$ will be updated by adding $Q(0)$ that has been harvested in the previous $T$ period.

To summarize, at each transmission phase, the choice of $K$ is done such that

$$
K= \begin{cases}N_{p}, & \text { if } E_{t} \leq 0, \\ k, & \text { if }(14) \text { is satisfied for } k=1, \ldots, N_{p}-1, \\ 0, & \text { if } Q(1)<E_{t}\end{cases}
$$

\section{B. Probability Mass Function (PMF) of $K$}

As shown in the EH model explained above, the number of selected antennas $K$ is random and depends on the value of $E_{t}$ and the channels $h_{p j}$ and $h_{s j}$. Hence, we need to derive its PMF. Also, the expression of the PMF of $K$ will serve as a preliminary result that we need to analyze the performance of our system model. Based on the $\mathrm{EH}$ model explained above, the PMF of $K$ can be written, for a given $E_{t}>0$, as

$$
\mathcal{P}_{K}(k)= \begin{cases}0, & \text { if } k=N_{p} \\ 1-\mathcal{F}^{(1)}\left(E_{t}\right), & \text { if } k=N_{p}-1, \\ \int_{0}^{E_{t}} f^{\left(N_{p}-k-1\right)}(y)\left(1-\mathcal{F}^{(1)}\left(E_{t}-y\right)\right) \mathrm{d} y, & \text { if } 1 \leq k \leq N_{p}-2, \\ \mathcal{F}^{\left(N_{p}-1\right)}\left(E_{t}\right), & \text { if } k=0 .\end{cases}
$$

Before going forward, we notice that if the value of the threshold $E_{t}$ is very large, it is highly probable for $K$ to be equal to zero, however if it is very small and positive, it is more probable for $K$ to be equal to $N_{p}-1$. Now, let us present the exact expression of the PMF of $K$ for Rayleigh fading channels.

Theorem 1: Based on the EH model explained above, for a given $E_{t}>0$, the PMF of $K$ is expressed, depending on the value of $\varepsilon_{p s}$ either zero or not.

- If $\varepsilon_{p s}=0$, the PMF of $K$ is given by

$$
\mathcal{P}_{K}(k)= \begin{cases}0, & \text { if } k=N_{p}, \\ \frac{\left(\frac{E_{t}}{\zeta \varepsilon_{s}}\right)^{2\left(N_{p}-k-1\right)}\left(1+\frac{1}{2\left(N_{p}-k\right)-1} \frac{E_{t}}{\zeta \varepsilon_{s}}\right)}{\Gamma\left(2\left(N_{p}-k\right)-1\right)} e^{-\frac{E_{t}}{\zeta \varepsilon_{s}},}, & \text { if } 1 \leq k \leq N_{p}-1, \\ \frac{\gamma\left(2\left(N_{p}-1\right) \frac{E_{t}}{\zeta \varepsilon_{s}}\right)}{\Gamma\left(2\left(N_{p}-1\right)\right)}, & \text { if } k=0 .\end{cases}
$$

- If $\varepsilon_{p s} \neq 0$, the PMF of $K$ is given by

$$
\mathcal{P}_{K}(k)= \begin{cases}0, & \text { if } k=N_{p}, \\ \frac{\varepsilon_{p} e^{-\frac{E_{t}}{\zeta \varepsilon_{p}}}-\varepsilon_{s} e^{-\frac{E_{t}}{\zeta \varepsilon_{s}}}}{\varepsilon_{p s}}, & \text { if } k=N_{p}-1, \\ \varphi_{k}\left(E_{t}, E_{t}\right), & \text { if } 1 \leq k \leq N_{p}-2, \\ \frac{\gamma\left(N_{p}-1, \frac{E_{t}}{\zeta \varepsilon_{p}}\right)}{\Gamma\left(N_{p}-1\right)}-\psi_{1}\left(E_{t}\right), & \text { if } k=0,\end{cases}
$$




$$
\begin{aligned}
\varphi_{n}(x, y) & =\frac{\sqrt{\pi \zeta^{2} \varepsilon_{s} \varepsilon_{p}} x^{N_{p}-n-\frac{3}{2}} e^{-\left(\frac{1}{\varepsilon_{s}}+\frac{1}{\varepsilon_{p}}\right) \frac{x}{2 \zeta}}}{\Gamma\left(N_{p}-n\right)\left(\zeta \varepsilon_{p s}\right)^{N_{p}-n-\frac{1}{2}}}\left[\left(\frac{\varepsilon_{p} e^{-\frac{y-x}{\zeta \varepsilon_{p}}}-\varepsilon_{s} e^{-\frac{y-x}{\zeta \varepsilon_{s}}}}{\varepsilon_{p s}}\left(2\left(N_{p}-n\right)-1\right)+\frac{\varepsilon_{p} e^{-\frac{y-x}{\zeta \varepsilon_{p}}}+\varepsilon_{s} e^{-\frac{y-x}{\zeta \varepsilon_{s}}}}{\varepsilon_{s} \varepsilon_{p}} \frac{x}{2 \zeta}\right) I_{N_{p}-n-\frac{1}{2}}\left(\frac{\varepsilon_{p s}}{\varepsilon_{s} \varepsilon_{p}} \frac{x}{2 \zeta}\right)\right. \\
& \left.+\frac{\varepsilon_{p} e^{-\frac{y-x}{\zeta \varepsilon_{p}}}-\varepsilon_{s} e^{-\frac{y-x}{\zeta \varepsilon_{s}}}}{\varepsilon_{s} \varepsilon_{p}} \frac{x}{2 \zeta} I_{N_{p}-n+\frac{1}{2}}\left(\frac{\varepsilon_{p s}}{\varepsilon_{s} \varepsilon_{p}} \frac{x}{2 \zeta}\right)\right] .
\end{aligned}
$$

where $\varphi_{n}(x, y)$ is defined in (19) for $0 \leq n \leq N_{p}-2$, and $0<x \leq y$.

Proof: The proof is derived in Appendix C.

Remark 2: Note that, if $E_{t} \leq 0$, the probability that $K=N_{p}$ is always equal to one, while it is zero otherwise. In other words, the PMF of $K$ reduces to

$$
\mathcal{P}_{K}(k)= \begin{cases}1, & \text { if } k=N_{p}, \\ 0, & \text { if } k=0, \ldots, N_{p}-1 .\end{cases}
$$

Moreover, if the value of $E_{t}$ is very large, it is highly probable that all antennas are used to harvest the energy and $K$ is equal to zero. Also, if the value of $E_{t}$ is very small and positive, it is highly probable that most antennas are used to decode the information data and $K$ is equal to $N_{p}-1$.

\section{Performance Analysis}

In this section, we study the performance analysis of the described scheme. Since we have a SWIPT-constrained system, we need to address the energy and data metrics in order to have a full picture of the performance of our system. Usually, there is a tradeoff between the harvested energy and the decoded data [3], [8]. We inquiry if we still have the same scenario in our model. So, we propose to derive the energy metrics such as the average harvested energy and the power outage at PR, and the data metrics such as the primary data outage probability, the secondary data outage probability, and the mutual outage probability (MOP) that was first introduced in [26].

\section{A. Average Harvested Energy at PR}

The average harvested energy at PR is defined as the sum of average harvested energy for each $K=0, \ldots, N_{p}-1$.

$$
\bar{Q}=\mathbb{E}[Q]=\sum_{k=0}^{N_{p}-1} \mathbb{E}[Q(k) \mid K=k] \mathcal{P}_{K}(k) .
$$

Since the PT-PR channels and ST-PR channels are independent and identically distributed, we can show that the average harvested energy is given by

$$
\bar{Q}=\zeta\left(\varepsilon_{p}+\varepsilon_{s}\right)\left[N_{p}-\sum_{k=1}^{N_{p}-1} k \mathcal{P}_{K}(k)\right] .
$$

Since the PMF of $K$ depends on the value of $\varepsilon_{p s}$, the average harvested energy at PT depends also on the value of $\varepsilon_{p s}$ as in (23).

\section{B. Power Outage at $P R$}

The power outage at PR is defined as the probability that the harvested energy is below a certain power threshold $\gamma_{q}$. Since the harvested energy depends on the selection of the number of antennas $K$, so, the power outage is the sum of the power outage for each $k=0, \ldots, N_{p}-1$.

$$
\mathcal{P} O=\sum_{k=0}^{N_{p}-1} \mathcal{P} O(k)=\sum_{k=0}^{N_{p}-1} \mathcal{P}\left(\mathcal{Q}(k) \leq \gamma_{q} \& K=k\right) .
$$

Considering the explained EH model in Section III, we show that the power outage at PR is expressed depending on $\gamma_{q}-E_{t}$ either less or greater than zero.

$$
\mathcal{P} O= \begin{cases}\int_{0}^{\gamma_{q}} f^{\left(N_{p}-1\right)}(y) \mathcal{F}^{(1)}\left(\gamma_{q}-y\right) \mathrm{d} y, & \text { if } \gamma_{q}<E_{t}, \\ \mathcal{F}^{(1)}\left(\gamma_{q}\right)-\sum_{k=0}^{N_{p}-2} \int_{0}^{E_{t}} f^{\left(N_{p}-k-1\right)}(y) & \\ \times\left(1-\mathcal{F}^{(1)}\left(\gamma_{q}-y\right)\right) \mathrm{d} y, & \text { if } \gamma_{q} \geq E_{t} .\end{cases}
$$

The resolution of this expression depends on the value of $\varepsilon_{p s}$ either zero or not.

$$
\begin{aligned}
& \text { - If } \varepsilon_{p s}=0, \\
& \qquad \mathcal{P O}= \begin{cases}\frac{\gamma\left(2 N_{p}, \frac{\gamma q}{\zeta \varepsilon_{s}}\right)}{\Gamma\left(2 N_{p}\right)}, & \text { if } \gamma_{q}<E_{t}, \\
1-\sum_{k=0}^{N_{p}-1} \frac{\left(1+\frac{\gamma_{q}}{\zeta \varepsilon_{s}}-\frac{E_{t}}{\zeta \varepsilon_{s}} \frac{2\left(N_{p}-k-1\right)}{2\left(N_{p}-k\right)-1}\right)\left(\frac{E_{t}}{\zeta \varepsilon_{s}}\right)^{2\left(N_{p}-k-1\right)} e^{-\frac{\gamma_{q}}{\zeta \varepsilon_{s}}}}{\Gamma\left(2\left(N_{p}-k\right)-1\right)}, & \text { if } \gamma_{q} \geq E_{t} .\end{cases}
\end{aligned}
$$

- If $\varepsilon_{p s} \neq 0$, the power outage at PR is expressed as in (27).

\section{Mutual Outage Probability (MOP)}

In addition, the MOP is a metric that declares a data outage event when either the PR or SR is in an outage. The MOP expression is given by

$$
\mathcal{M O P}=\mathcal{P}\left[\gamma_{I D}<\xi_{p} \text { or } \gamma_{S}<\xi_{s}\right]=\mathcal{P}_{P, \text { out }}\left(\xi_{p}\right)+\mathcal{P}_{S, \text { out }}\left(\xi_{s}\right)
$$

where $\xi_{p}=2^{R_{p}}-1, \xi_{s}=2^{R_{s}}-1$, and $R_{p}$ and $R_{s}$ are the transmission data rates at PT and ST, respectively. Note that the right hand side of (28) is obtained since $\gamma_{I D}$ and $\gamma_{S}$ are independent. In order to derive the expression of MOP, we need first to derive the primary data outage probability and the secondary data outage probability.

First, the primary data outage probability is expressed depending on the sign of $E_{t}$ and the possible values of $K$ for a given $E_{t}$. If $E_{t}$ is negative, the data outage at PR happens when $\gamma_{I D}\left(N_{p}\right)$ is less or equal to $\xi_{p}$. However, if $E_{t}>0$, the data outage at PR depends on the value of $K$. If $K=0$, it is certain that PR is in data outage since all receiving antennas are used to harvest the energy. If $K=1, \ldots, N_{p}-1$, the data outage at PR happens when $\gamma_{I D}(K)$ is less or equal to $\xi_{p}$. 


$$
\overline{\boldsymbol{Q}}= \begin{cases}\zeta\left(\varepsilon_{p}+\varepsilon_{s}\right)\left[N_{p}-\sum_{k=1}^{N_{p}-1} \frac{k\left(\frac{E_{t}}{\zeta \varepsilon_{s}}\right)^{2\left(N_{p}-k-1\right)}\left(1+\frac{1}{2\left(N_{p}-k\right)-1} \frac{E_{t}}{\zeta \varepsilon_{s}}\right)}{\Gamma\left(2\left(N_{p}-k\right)-1\right)} e^{-\frac{E_{t}}{\zeta \varepsilon_{s}}}\right], & \text { if } \varepsilon_{p s}=0 \\ \zeta\left(\varepsilon_{p}+\varepsilon_{s}\right)\left[N_{p}-\left(N_{p}-1\right)\left(\frac{\varepsilon_{p} e^{-\frac{E_{t}}{\zeta \varepsilon_{p}}}-\varepsilon_{s} e^{-\frac{E_{t}}{\zeta \varepsilon_{s}}}}{\varepsilon_{p s}}\right)\right], & \text { if } \varepsilon_{p s} \neq 0\end{cases}
$$

$$
\mathcal{P O}= \begin{cases}\frac{\gamma\left(N_{p}-1, \frac{\gamma_{q}}{\zeta q_{p}}\right)}{\Gamma\left(N_{p}-1\right)}-\psi_{1}\left(\gamma_{q}\right)-\varphi_{0}\left(\gamma_{q}, \gamma_{q}\right), & \text { if } \gamma_{q}<E_{t} \\ 1-\frac{\varepsilon_{p} e^{-\frac{\gamma_{q}}{\zeta \varepsilon_{p}}}-\varepsilon_{s} e^{-\frac{\gamma_{q}}{\zeta \varepsilon_{s}}}}{\varepsilon_{p s}}-\sum_{k=0}^{N_{p}-2} \varphi_{k}\left(E_{t}, \gamma_{q}\right), & \text { if } \gamma_{q} \geq E_{t}\end{cases}
$$

Subsequently, we can write

$\mathcal{P}_{P, \text { out }}\left(\xi_{p}\right)= \begin{cases}\mathcal{F}_{\gamma_{I D}\left(N_{p}\right)}\left(\xi_{p}\right), & \text { if } E_{t} \leq 0, \\ \sum_{k=1}^{N_{p}-1} \mathcal{F}_{\gamma_{I D}(K)}\left(\xi_{p}\right) \mathcal{P}_{K}(k)+\mathcal{P}_{K}(0), & \text { if } E_{t}>0,\end{cases}$

where $\mathcal{F}_{\gamma_{I D}(K)}(\cdot)$ is the conditional CDF of SINR at PR for a given $1 \leq K \leq N_{p}$, and $\mathcal{P}_{K}(\cdot)$ is the PMF of $K$ derived in Theorem 1 . To explicitly express the data outage probability at PR, we need to derive the conditional CDF of SINR at PR $\mathcal{F}_{\gamma_{I D}(K)}(\cdot)$, for $K=1, \ldots, N_{p}$, besides to the PMF of $K$.

After mathematical manipulations, we show that the conditional CDF of $\gamma_{I D}(K)$ is given in the following theorem:

Theorem 2: For a given $1 \leq K \leq N_{p}$, the conditional CDF of $\gamma_{I D}(K)$ is written as

$$
\begin{aligned}
\mathcal{F}_{\gamma_{I D}(K)}\left(\xi_{p}\right) & =\frac{1}{\Gamma(K)}\left(\gamma\left(K, \frac{\xi_{p}}{\beta_{1}}\right)\right. \\
& \left.+e^{\frac{1}{\beta_{2}}}\left(1+\frac{1}{\xi_{p}} \frac{\beta_{1}}{\beta_{2}}\right)^{-K} \Gamma\left(K,\left(1+\frac{1}{\xi_{p}} \frac{\beta_{1}}{\beta_{2}}\right) \frac{\xi_{p}}{\beta_{1}}\right)\right) .
\end{aligned}
$$

Proof: The proof is derived in Appendix D.

Subsequently, the primary data outage probability can be deduced by substituting the results of Theorem 1 and Theorem 2 in (29) to obtain (31).

Then, the secondary data outage probability is expressed in the following theorem.

Theorem 3: The secondary data outage probability is expressed as in (32), where $p_{0}\left(\xi_{s}\right)=\frac{\xi_{s} \alpha_{2}}{\alpha_{1}+\xi_{s} \alpha_{2}}$.

Proof: The secondary data outage probability is written as

$\mathcal{P}_{S, \text { out }}\left(\xi_{s}\right)=\int_{\xi_{s}}^{\infty}\left(1-\mathcal{F}_{\gamma_{p d} \mid u}\left(\frac{u}{\xi_{s}}-1\right)\right) f_{\gamma_{s d}}(u) \mathrm{d} u+\int_{0}^{\xi_{s}} f_{\gamma_{s d}}(u) \mathrm{d} u$

Given that $\mathcal{F}_{\gamma_{p d}}(u)=1-e^{-\frac{u}{\alpha_{2}}}$, we can show that the expression of the CDF of $\gamma_{S}$ is written as

$$
\begin{gathered}
\mathcal{P}_{S, \text { out }}\left(\xi_{s}\right)=\underbrace{\mathcal{F}_{\gamma_{s d}}\left(\xi_{s}\right)}_{I_{1}\left(\xi_{s}\right)}+e^{\frac{1}{\alpha_{2}}} \underbrace{\int_{\xi_{s}}^{\infty} e^{-\frac{u}{\xi_{s} \alpha_{2}}} f_{\gamma_{s d}}(u) \mathrm{d} u}_{I_{2}\left(\xi_{s}\right)} .
\end{gathered}
$$

Then, we express $\mathcal{I}_{1}\left(\xi_{s}\right)$ using the expression of $\mathcal{F}_{\gamma_{s d}}(y)$ in Lemma 1 , and we use the expression of $\mathcal{I}_{2}\left(\xi_{s}\right)$ from (112) in Appendix $E$ to deduce the expression of the secondary data outage probability in (32).

Finally, to get the expression of the MOP metric, we just substitute the expression of (31) and (32) in (28).

\section{Special Cases and Asymptotic Results}

In this part, we will address some special cases to show the relevance of our analysis and some asymptotic results of the performance metrics derived in Section IV.

\section{A. Single Antenna at ST}

The first special case to consider is when the ST has a single transmit antenna. In our analysis, the ST has multiple antennas, but only one antenna is selected to transmit. What if we have only one antenna at ST from the beginning. In this case, the performance analysis in terms of the secondary data outage probability is different and simpler. The statistics of $\gamma_{s d}$ is no more given by Lemma 1 and is simply following the Rayleigh distribution. In that case, the data outage probability at SR simplifies to

$$
\mathcal{P}_{S, \text { out }}\left(\xi_{s}\right)=1-\left(1-p_{0}\left(\xi_{s}\right)\right) e^{-\frac{\xi_{s}}{\alpha_{1}}} .
$$

All the other performance metrics still hold.

\section{B. Maximum Channel Gain Antenna Selection at ST}

In our analysis, we select the transmit antenna at ST that maximizes the channel gain while minimizing the global effect of interference at PR. The condition of minimal interference at PR makes the selection criteria complicated and requires the CSI knowledge between ST and PR which is hard to obtain perfectly in practice. What if we drop this minimal interference condition and look only to maximize the channel gain between ST and SR? In the Rayleigh fading channels, according to the work done in [29], the maximum channel gain antenna selection perfectly approximates our selection scheme. In other fading channels, the maximum channel gain antenna selection is just an approximation of our selection scheme. In general, the maximum channel gain antenna selection allows better data outage probability at SR, but higher destructive interference at PR. Our selection scheme worsens the data outage probability at $\mathrm{SR}$ at the expense of protecting PR from the interference. So, let us investigate this tight approximation of our selection scheme. In this case, the PDF and CDF of $\gamma_{s d}$ simplifies to

$$
\begin{aligned}
& f_{\gamma_{s d}}(x)=\frac{N_{s}}{\alpha_{1}} e^{-\frac{x}{\alpha_{1}}}\left(1-e^{-\frac{x}{\alpha_{1}}}\right)^{N_{s}-1}, \\
& \mathcal{F}_{\gamma_{s d}}(y)=\left(1-e^{-\frac{y}{\alpha_{1}}}\right)^{N_{s}},
\end{aligned}
$$

where the selected antenna satisfies

$$
s=\underset{1 \leq l \leq N_{s}}{\arg \max }\left|h_{l d}\right|^{2} .
$$




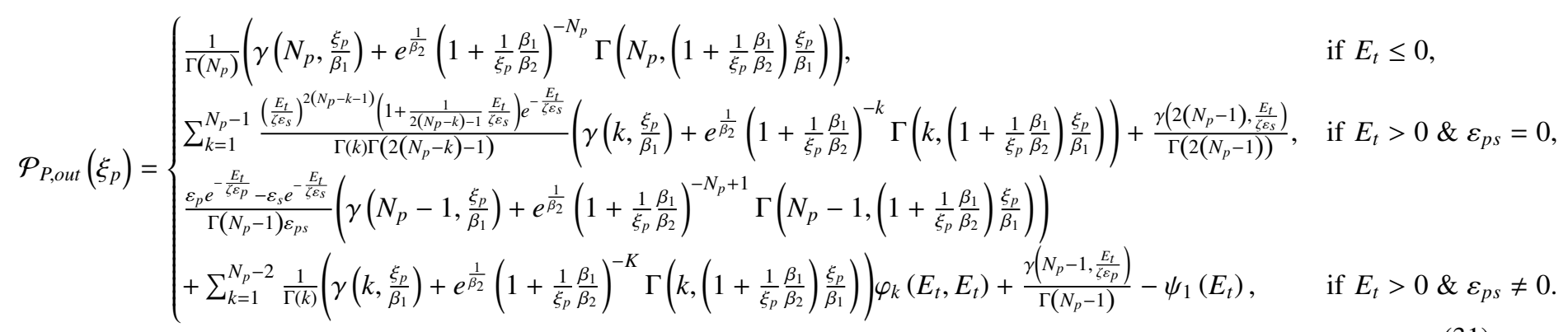

$$
\begin{aligned}
\mathcal{P}_{S, \text { out }}\left(\xi_{s}\right) & =N_{s}\left(1-\left(1-p_{0}\left(\xi_{s}\right)\right) e^{-\frac{\xi_{s}}{\alpha_{1}}}\right)+\frac{N_{s}}{\Gamma\left(N_{p}\right)} \sum_{j=1}^{N_{s}-1}\left(\begin{array}{c}
N_{s}-1 \\
j
\end{array}\right)\left[\sum _ { i = 0 } ^ { N _ { p } ( j + 1 ) - 1 } ( \begin{array} { c } 
{ N _ { p } ( j + 1 ) - 1 } \\
{ i }
\end{array} ) \frac { ( - 1 ) ^ { j + i } } { ( i + 1 ) \alpha _ { 1 } } \left[\gamma\left(N_{p}+1, \frac{y}{\alpha_{1}}\right)\right.\right. \\
& \left.+\alpha_{1}\left(\frac{y}{\alpha_{1}}\right)^{i+1} \Gamma\left(N_{p}-i, \frac{y}{\alpha_{1}}\right)\right]+e^{\frac{1}{\alpha_{2}}} \sum_{i=0}^{N_{p}-1}\left(\begin{array}{c}
N_{p}(j+1)-1 \\
i
\end{array}\right)(-1)^{j+i} \Gamma\left(N_{p}-i\right) \sum_{l=i+1}^{N_{p}} \frac{\left(p_{0}\left(\xi_{s}\right)\right)^{l} \Gamma\left(l, \frac{\xi_{s}}{\alpha_{1}\left(\xi_{s}\right)}\right)}{\Gamma(l-i)} \\
& +\sum_{i=N_{p}}^{N_{p}(j+1)-1}\left(\begin{array}{c}
N_{p}(j+1)-1 \\
i
\end{array}\right) \frac{(-1)^{j+N_{p}}\left(\frac{\xi_{s} \alpha_{2}}{\alpha_{1}}\right)^{i+1}}{\Gamma\left(i-N_{p}+1\right)}\left(\Gamma\left(i+1, \frac{1}{\alpha_{2}}\right) \Gamma\left(0, \frac{\xi_{s}}{\alpha_{1}}\right)-\Gamma(i+1) \sum_{l=0}^{i} \frac{\left(\frac{\alpha_{1} p_{0}\left(\xi_{s}\right)}{\xi_{s} \alpha_{2}}\right)^{l} \Gamma\left(l, \frac{\xi_{s}}{\alpha_{1} p_{0}\left(\xi_{s}\right)}\right)}{\Gamma(l+1)}\right) \\
& \left.-\sum_{i=N_{p}+1}^{N_{p}(j+1)-1}\left(\begin{array}{c}
N_{p}(j+1)-1 \\
i
\end{array}\right) \frac{(-1)^{j+N_{p}}}{\Gamma\left(i-N_{p}+1\right)} \sum_{l=N_{p}+1}^{i+1}(-1)^{i-l} \Gamma(i-l+1)\left(p_{0}\left(\xi_{s}\right)\right)^{l} \Gamma\left(l, \frac{\xi_{s}}{\alpha_{1} p_{0}\left(\xi_{s}\right)}\right)\right] .
\end{aligned}
$$

Here, the PDF and CDF of $\gamma_{s d}$ only depend on $N_{s}$, compared to Lemma 1 where the PDF and CDF of $\gamma_{s d}$ depend on both $N_{s}$ and $N_{p}$, for $x \geq 0, y \geq 0$. Given these statistics of $\gamma_{s d}$, the secondary data outage probability is given by

$$
\mathcal{P}_{S, \text { out }}\left(\xi_{s}\right)=\sum_{j=0}^{N_{s}}\left(\begin{array}{c}
N_{s} \\
j
\end{array}\right)(-1)^{j} \frac{e^{-\frac{j \xi_{s}}{\alpha_{1}}}}{1+j \xi_{s} \frac{\alpha_{2}}{\alpha_{1}}} .
$$

\section{Absence of Secondary Transmission}

Moreover, to validate the relevance of our model, we should compare it to the case when the ST is not present. This comparison will show that the presence of ST is beneficial to both ends. If ST is not present, the combined SNR at PR is given by

$$
\gamma_{I D}^{0}\left(K_{0}\right)=\frac{P_{p}}{\sigma_{p}^{2}} \sum_{j=1}^{K_{0}}\left|h_{p j}\right|^{2},
$$

and the harvested energy at PR is given by

$$
Q^{0}\left(K_{0}\right)= \begin{cases}\sum_{j=K_{0}+1}^{N_{p}} E_{j}^{0}, & \text { if } 0 \leq K_{0}<N_{p}, \\ 0, & \text { if } K_{0}=N_{p},\end{cases}
$$

where $K_{0}$ is the number of antennas selected at PR to decode the data when ST is not present, and $E_{j}^{0}=\zeta T P_{p}\left|h_{p j}\right|^{2}$ is the harvested energy at the $\mathrm{j}$ 'th antenna of PR. Here, the PDF and $\mathrm{CDF}$ of $Q^{0}\left(K_{0}\right)$ are given by

$$
\begin{aligned}
f_{0}^{\left(N_{p}-k_{0}\right)}\left(z_{0}\right) & =\frac{1}{\Gamma\left(N_{p}-k_{0}\right)} \frac{z_{0}^{N_{p}-k_{0}-1}}{\left(\zeta \varepsilon_{p}\right)^{N_{p}-k_{0}}} e^{-\frac{z_{0}}{\zeta \varepsilon_{p}}}, \\
\mathcal{F}_{0}^{\left(N_{p}-k_{0}\right)}\left(t_{0}\right) & =\frac{\gamma\left(N_{p}-k_{0}, \frac{t_{0}}{\zeta \varepsilon_{p}}\right)}{\Gamma\left(N_{p}-k_{0}\right)},
\end{aligned}
$$

respectively, for $k_{0}=0, \ldots, N_{p}-1, z_{0} \geq 0$, and $t_{0} \geq 0$. The selection of the number of antennas $K_{0}$ at PR is only based on the harvested energy from PT transmissions. The same EH model discussed in Section III applies here and the PMF of $K_{0}$, for a given $E_{t}>0$, is given by

$$
\mathcal{P}_{K_{0}}\left(k_{0}\right)= \begin{cases}0, & \text { if } k_{0}=N_{p}, \\ \frac{1}{\Gamma\left(N_{p}-k_{0}\right)}\left(\frac{E_{t}}{\zeta \varepsilon_{p}}\right)^{N_{p}-k_{0}-1} e^{-\frac{E_{t}}{\zeta \varepsilon_{p}},} & \text { if } 1 \leq k_{0} \leq N_{p}-1, \\ \frac{1}{\Gamma\left(N_{p}-1\right)} \gamma\left(N_{p}-1, \frac{E_{t}}{\zeta \varepsilon_{p}}\right), & \text { if } k_{0}=0 .\end{cases}
$$

The same Remark 2 applies here when $E_{t} \leq 0$. Moreover, the average harvested energy at $\mathrm{PR}$ is given by

$$
\begin{aligned}
\bar{Q}^{(0)} & =\frac{\zeta \varepsilon_{p}}{\Gamma\left(N_{p}-1\right)}\left[N_{p} \gamma\left(N_{p}-1, \frac{E_{t}}{\zeta \varepsilon_{p}}\right)+\left(1+\frac{E_{t}}{\zeta \varepsilon_{p}}\right) \Gamma\left(N_{p}-1, \frac{E_{t}}{\zeta \varepsilon_{p}}\right)\right. \\
& \left.-\left(\frac{E_{t}}{\zeta \varepsilon_{p}}\right)^{N_{p}-1} e^{-\frac{E_{t}}{\zeta \varepsilon_{p}}}\right]
\end{aligned}
$$

which was obtained using $[28,(8.352 .4)]$. In addition, the power outage in the absence of ST is obtained in a similar way as in (25) and it can be shown that it is given by

$$
\mathcal{P} O^{(0)}= \begin{cases}\frac{\gamma\left(N_{p}, \frac{\gamma_{q}}{\zeta \varepsilon_{p}}\right)}{\Gamma\left(N_{p}\right)}, & \text { if } \gamma_{q}<E_{t}, \\ 1-\left(1-\frac{\gamma\left(N_{p}, \frac{E_{t}}{\zeta p_{p}}\right)}{\Gamma\left(N_{p}\right)}\right) e^{-\frac{\gamma_{q}-E_{t}}{\zeta \varepsilon_{p}}}, & \text { if } \gamma_{q} \geq E_{t} .\end{cases}
$$

Finally, the conditional data outage probability at PR, for a given $K_{0}=1, \ldots, N_{p}$, in the absence of $\mathrm{ST}$ is given by

$$
\mathcal{F}_{\gamma_{I D}^{0}\left(K_{0}\right)}\left(\xi_{p}\right)=\frac{1}{\Gamma\left(K_{0}\right)} \gamma\left(K_{0}, \frac{\xi_{p}}{\beta_{1}}\right) .
$$


Subsequently, the data outage probability at PR is given by

$$
\mathcal{P}_{P, \text { out }}^{(0)}\left(\xi_{p}\right)= \begin{cases}\frac{1}{\Gamma\left(N_{p}\right)} \gamma\left(N_{p}, \frac{\xi_{p}}{\beta_{1}}\right), & \text { if } E_{t} \leq 0, \\ \sum_{k_{0}=1}^{N_{p}-1} \frac{\left(\frac{E_{t}}{\zeta \varepsilon_{p}}\right)^{N_{p}-k_{0}-1} e^{-\frac{E_{t}}{\zeta \varepsilon_{p}}}}{\Gamma\left(k_{0}\right) \Gamma\left(N_{p}-k_{0}\right)} \gamma\left(k_{0}, \frac{\xi_{p}}{\beta_{1}}\right) & \\ +\frac{1}{\Gamma\left(N_{p}-1\right)} \gamma\left(N_{p}-1, \frac{E_{t}}{\zeta \varepsilon_{p}}\right), & \text { if } E_{t}>0 .\end{cases}
$$

The validity of our model is verified whenever we have that the average harvested energy, the power outage and the data outage probability at PR improves in the presence of ST.

\section{Time Switching (TS) Scheme at PR}

Finally, to support our choice of AS scheme at PR, we compare our results to the case where PR uses the TS scheme to separate the EH and ID operations over the time. Let $\tau \in$ $[0,1]$ be the time ratio needed to harvest enough energy and $(1-\tau)$ be the time needed to decode the information data at PR. During the EH period, all antennas are used to harvest the energy, while during the ID period, all the antennas are used to decode the information data. For a given $\tau$, the harvested energy at $\mathrm{PR}$ is given by

$$
Q_{T S}=\tau Q(0),
$$

where $\tau$ should satisfy

$$
\tau=\min \left(\frac{E_{t}}{Q(0)}, 1\right),
$$

in order to harvest an amount of energy no more than the energy requirement $E_{t}$ at PR. For that, the primary data outage probability is equal to $\mathcal{F}_{\gamma_{I D}\left(N_{p}\right)}\left(\xi_{p}^{T S}\right)$ given in Theorem 2 as

$$
\begin{aligned}
\mathcal{P}_{P, \text { out }}^{T S}\left(\xi_{p}^{T S}\right) & =\frac{1}{\Gamma\left(N_{p}\right)}\left(\gamma\left(N_{p}, \frac{\xi_{p}^{T S}}{\beta_{1}}\right)\right. \\
& +e^{\frac{1}{\beta_{2}}}\left(1+\frac{1}{\xi_{p}^{T S}} \frac{\beta_{1}}{\beta_{2}}\right)^{-N_{p}} \Gamma\left(N_{p},\left(1+\frac{1}{\xi_{p}^{T S}} \frac{\beta_{1}}{\beta_{2}} \frac{\xi_{p}^{T S}}{\beta_{1}}\right)\right),
\end{aligned}
$$

where $\xi_{p}^{T S}$ depends on both the time ratio $\tau$ and the transmission rate $R_{p}$ and is defined in a way to have $R_{p}=$ $(1-\tau) \log _{2}\left(\xi_{p}^{T S}+1\right)$. Following that, the average harvested energy and the power outage for the TS scheme are given by

$$
\begin{aligned}
\bar{Q}^{T S} & =E_{t}\left(1-\mathcal{F}^{\left(N_{p}\right)}\left(E_{t}\right)\right)+\int_{0}^{E_{t}} q f^{\left(N_{p}\right)}(q) \mathrm{d} q, \\
\mathcal{P} O^{T S} & = \begin{cases}\mathcal{F}^{\left(N_{p}\right)}\left(\gamma_{q}\right), & \text { if } \gamma_{q}<E_{t}, \\
1, & \text { if } \gamma_{q} \geq E_{t} .\end{cases}
\end{aligned}
$$

But, if ST is not present, all the performance metrics relevant to the TS scheme simplify to

$$
\begin{aligned}
\mathcal{P}_{P, \text { out }}^{0, T S}\left(\xi_{p}^{T S}\right) & =\frac{\gamma\left(N_{p}, \frac{\xi_{p}^{T S}}{\beta_{1}}\right)}{\Gamma\left(N_{p}\right)}, \\
\overline{\boldsymbol{Q}}^{0, T S} & =\frac{E_{t} \Gamma\left(N_{p}, \frac{E_{t}}{\zeta \varepsilon_{p}}\right)+\zeta \varepsilon_{p} \gamma\left(N_{p}+1, \frac{E_{t}}{\zeta \varepsilon_{p}}\right)}{\Gamma\left(N_{p}\right)}, \\
\mathcal{P} O^{0, T S} & = \begin{cases}\frac{\gamma\left(N_{p}, \frac{\gamma_{q}}{\zeta \xi_{p}}\right)}{\Gamma\left(N_{p}\right)}, & \text { if } \gamma_{q}<E_{t}, \\
1, & \text { if } \gamma_{q} \geq E_{t} .\end{cases}
\end{aligned}
$$

Finally, please note that the TS scheme is limited in terms of the maximum average harvested energy and the transmission rate. The TS scheme cannot harvest more than $E_{t}$. Moreover, if $\tau=1$, the primary data outage probability is equal to 1 .

\section{E. Asymptotic Results}

In this part, we will investigate some asymptotic results of the performance metrics derived above.

- We have noted through the EH model that the value of $E_{t}$ has a direct impact on the PMF of $K$, which is itself has a direct impact on almost all the performance metrics studied, i.e. $\bar{Q}, \mathcal{P} O, \mathcal{P}_{P, o u t}$, and $\mathcal{M O P}$. So, let us address the two extreme cases of $E_{t}>0$, when $E_{t}$ goes to zero and when $E_{t}$ goes to infinity. Observing (15), we can see that

$$
\begin{aligned}
& K \underset{E_{t} \rightarrow 0}{\longrightarrow} N_{p}-1, \\
& K \underset{E_{t} \rightarrow \infty}{\longrightarrow} 0 .
\end{aligned}
$$

This result is important and will help us to derive the asymptotic results of all the metrics when $E_{t}$ goes to zero and when $E_{t}$ goes to infinity.

- If $E_{t} \rightarrow 0$, all the data and energy metrics $\mathcal{P}_{P, \text { out }}, \mathcal{P} O$, and $\bar{Q}$ reduce to

$$
\begin{aligned}
\bar{Q} & \approx \zeta\left(\varepsilon_{p}+\varepsilon_{s}\right), \\
\mathcal{P O} & \approx \mathcal{F}^{(1)}\left(\gamma_{q}\right), \\
\mathcal{P}_{P, \text { out }}\left(\xi_{p}\right) & \approx \mathcal{F}_{\gamma_{I D}\left(N_{p}-1\right)}\left(\xi_{p}\right) .
\end{aligned}
$$

- If $E_{t} \rightarrow \infty$, similarly, all the data and energy metrics $\mathcal{P}_{P, \text { out }}, \mathcal{P} O$, and $\bar{Q}$ reduce to

$$
\begin{aligned}
\bar{Q} & \approx N_{p} \zeta\left(\varepsilon_{p}+\varepsilon_{s}\right), \\
\mathcal{P} O & \approx \begin{cases}\frac{\gamma\left(2 N_{p}, \frac{\gamma_{q}}{\zeta \varepsilon_{s}}\right)}{\Gamma\left(2 N_{p}\right)}, & \text { if } \varepsilon_{p s}=0, \\
\frac{\gamma\left(N_{p}-1, \frac{\gamma q}{\zeta \varepsilon_{p}}\right)}{\Gamma\left(N_{p}-1\right)}-\psi_{1}\left(\gamma_{q}\right)-\varphi_{0}\left(\gamma_{q}, \gamma_{q}\right), & \text { if } \varepsilon_{p s} \neq 0,\end{cases}
\end{aligned}
$$

$$
\mathcal{P}_{P, \text { out }}\left(\xi_{p}\right) \approx 1
$$

- In Section V-C, we have discussed the special case where the ST is not present. The question that raises is what if PT is not present or what if $P_{p}$ or $P_{s}$ goes to infinity, how the performance of our system converges. So, here we propose to investigate the extreme cases where $P_{p}$ and $P_{s}$ go to zero and to infinity.

- First, when $P_{s}$ goes to zero, this asymptotic result is equivalent to the special case discussed above in Section V-C where ST is not present. The asymptotic results of $\bar{Q}, \mathcal{P O}$, and $\mathcal{P}_{P, \text { out }}$ are the same as the ones given in Section V-C, while the secondary data outage probability is obviously equal to one.

- However, when $P_{p}$ goes zero, the asymptotic results of $\bar{Q}$, and $\mathcal{P} O$ are the same as the ones in Section V-C, but after replacing $\varepsilon_{p}$ by $\varepsilon_{s}$. The asymptotic results of $\mathcal{P}_{S, \text { out }}$ reduces to $\mathcal{F}_{\gamma_{s d}}\left(\xi_{s}\right)$ and $\mathcal{P}_{P, \text { out }}$ goes to one, when $P_{p}$ goes to zero.

- But, if $P_{p}$ goes to infinity, the interference induced by PT at SR is very high and $\mathcal{P}_{S, \text { out }}$ goes to one. Also, 
as $P_{p}$ goes to infinity, $Q(K)$ goes also to infinity and, hence, according to (15), $K$ goes to $N_{p}-1$ and the asymptotic results of $\bar{Q}, \mathcal{P} O$, and $\mathcal{P}_{P, \text { out }}$ are the same as the ones discussed above when $E_{t}$ goes to zero. Applying the limit when $P_{p}$ goes to infinity to (59), (60), and (61), we get that $\bar{Q}, \mathcal{P} O$, and $\mathcal{P}_{P, \text { out }}$ converge to infinity, zero, and zero, respectively. Consequently, $\mathcal{M O P}$ goes to 1 , when $P_{p}$ goes to infinity.

- When $P_{s}$ goes to infinity, similarly, we show that $\bar{Q}$, $\mathcal{P} O, \mathcal{P}_{P, \text { out }}, \mathcal{P}_{P, \text { out }}$, and $\mathcal{M O P}$ go to infinity, zero, 1 , zero, and 1 , respectively.

\section{Numerical Results}

In this section, we present some simulation results to show the accuracy of the closed form expressions of the primary, secondary and mutual data outage probabilities, the power outage and the average harvested energy at PR derived in Section IV. We assume that the number of Monte Carlo (MC) simulation is $N_{\text {sim }}=10^{3}$. In all the figures, the MC simulations are referred to the none lined markers. The variances of the channels are chosen equal to $\lambda_{p p}=\lambda_{p d}=\lambda_{s d}=30$ $\mathrm{dBm}$. In order to have a fair comparison between schemes in terms of energy and data metrics, we consider the interference limited scenario where the noise variances at PR and SR are normalized to one. The conversion efficiency of the $\mathrm{EH}$ circuits at PR is $\zeta=60 \%$. In all figures, we take $\gamma_{q}$ equal to $30 \mathrm{dBm}$. In all the simulations, we verify the agreement between the analytic expressions and the Monte Carlo simulations.

\section{A. Number of Antennas $N_{p}$ and $N_{s}$ and Transmission Rates $R_{p}$ and $R_{S}$}

In Fig. 2, we have plotted the primary and secondary data outage probabilities versus the transmit power $P_{p}$ at PT, with different values of number of antennas at PR and ST and different values of transmission rates $R_{p}$ and $R_{s}$, for $P_{s}=30$ $\mathrm{dBm}, E_{p}=40 \mathrm{dBm}, E_{m}=20 \mathrm{dBm}$, and $E_{0}=610^{-4}$. We can see that the primary data outage probability decreases as we increase $P_{p}$, while the secondary data outage probability increases. Moreover, we can see that the primary data outage probability is independent of the number of antennas $N_{s}$ at ST which is accordance with the work done in [29], while it decreases as we increase the number of antennas $N_{p}$ at PR and we decrease the primary transmission rate $R_{p}$. In addition, we have compared our studied case to the case of absence of ST studied in V-C and to the case of TS scheme at PR in the presence of ST studied in Section V-D. We can observe first the gain due to the presence of ST in terms of the primary data outage probability especially for low $P_{p}$, while the presence of ST doesn't add much for high $P_{p}$. Moreover, we can see that the performance of the AS scheme and the TS scheme are almost the same for low $P_{p}$ while the TS scheme outperforms the AS scheme for high $P_{p}$, in terms of the primary data outage probability. On the other hand, the secondary data outage probability improves when the number of antennas $N_{s}$ and $N_{p}$ increases and the secondary transmission rate $R_{s}$ decreases. However, when we use the antenna selection explained in Section V-B, the secondary data outage probability improves
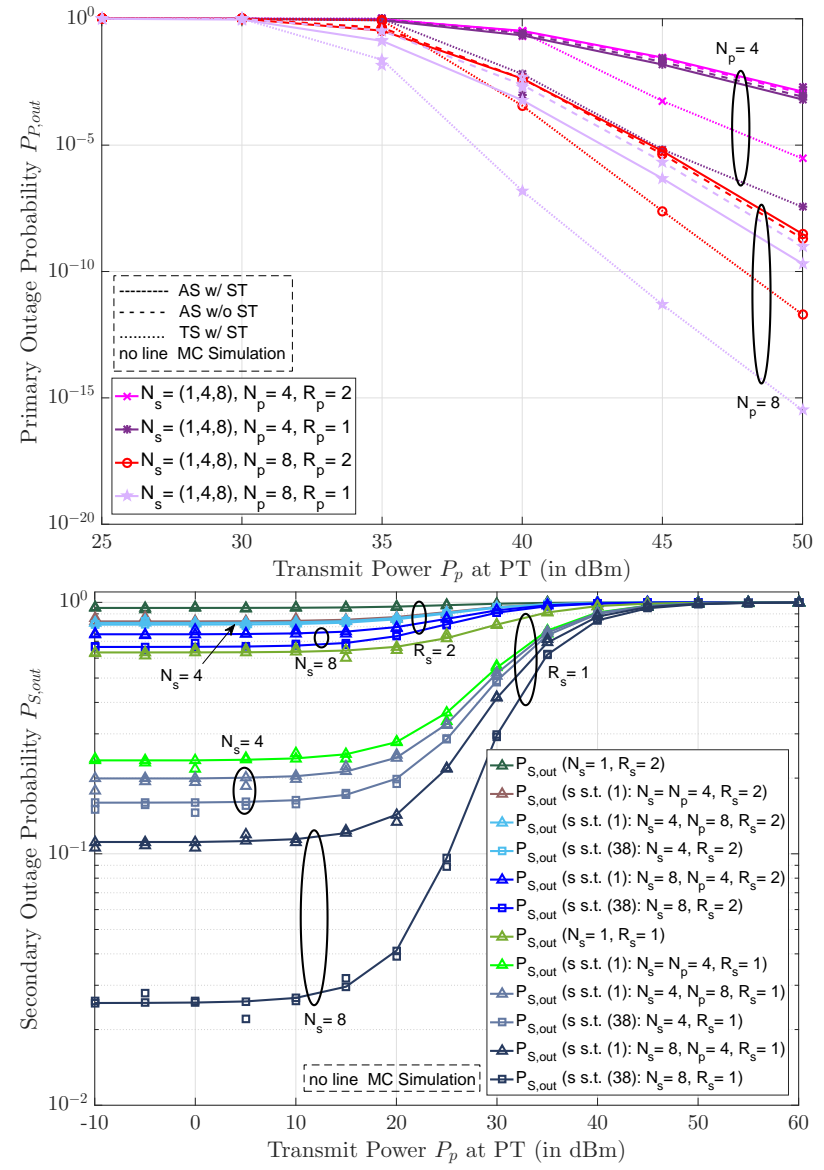

Figure 2. The primary and secondary data outage probabilities versus the transmit power $P_{p}$ at PT, with different values of number of antennas at PR and ST and different values of transmission rates $R_{p}$ and $R_{s}$, for $P_{s}=30$ $\mathrm{dBm}, E_{p}=40 \mathrm{dBm}, E_{m}=20 \mathrm{dBm}$, and $E_{0}=610^{-4}$.

when we increase $N_{s}$ and decrease $R_{s}$, independently of $N_{p}$. In addition, we can see that the secondary data outage probability when the selected antenna $s$ at ST is chosen as in (38) is always lower than the one when $s$ is chosen as in (1). This result is expected as explained in Section V-B.

In Figs. 3 and 4, we have plotted the mutual data outage probability $\mathcal{M O P}$ with different values of number of antennas $N_{s}$ and $N_{p}$ for transmission rates $R_{s}=R_{p}=2$, and with different values of transmission rates $R_{p}$ and $R_{s}$ for $N_{s}=4$ and $N_{p}=8$, respectively. The transmit power at ST is equal to $P_{s}=30 \mathrm{dBm}$. We have compared our analytic results of $\mathcal{M O P}$ to the case when we use the antenna selection scheme in (38) in Section V-C, to the case when we used the TS scheme in Section V-D, and to the Monte Carlo simulations. We can first see the agreement between all the analytic expressions and the Monte Carlo simulations. We can see also that the gain due to the use of the antenna selection scheme in (38) is only visible for low $P_{p}$. Moreover, we can see also that $\mathcal{M O P}$ increases for $P_{p}<30 \mathrm{dBm}$, and decreases for $P_{p} \geq 30$ $\mathrm{dBm}$ to converge to one for $P_{p}>50 \mathrm{dBm}$. For $P_{p}<30$ $\mathrm{dBm}, \mathcal{M O P}$ follows the performance of the secondary data outage probability and decreases as we increase the number of antennas $N_{s}$ as in Fig. 3 and as we decrease the transmission 


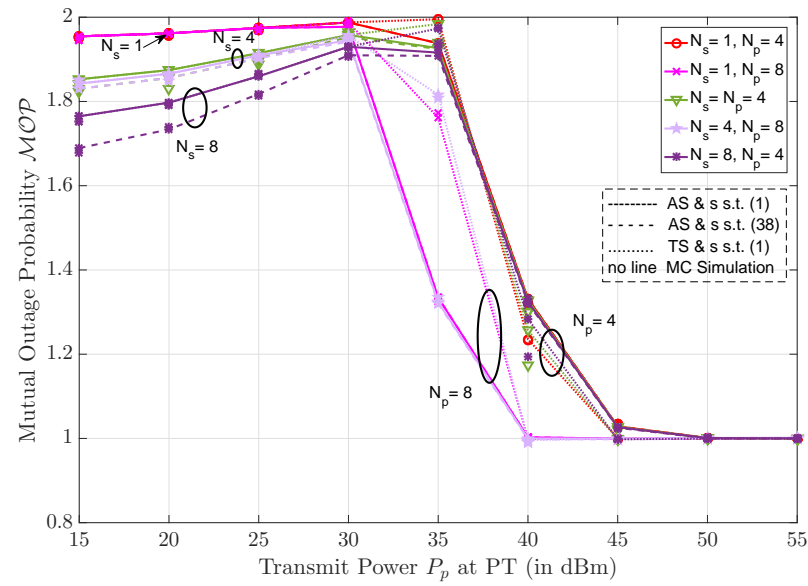

Figure 3. The mutual data outage probability $\mathcal{M O P}$ versus the transmit power $P_{p}$ at PT, with different values of number of antennas at PR and ST, for $R_{s}=R_{p}=2, P_{s}=30 \mathrm{dBm}, E_{p}=40 \mathrm{dBm}, E_{m}=20 \mathrm{dBm}$, and $E_{0}=610^{-4}$.

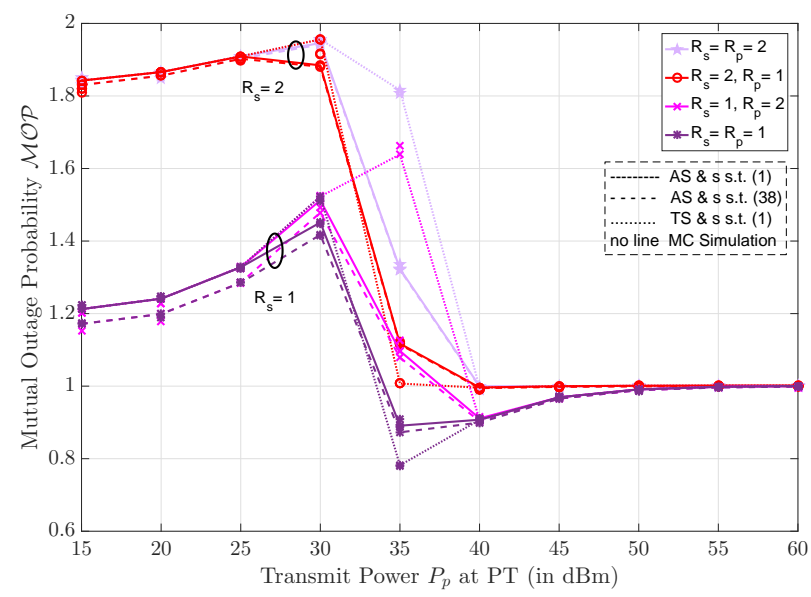

Figure 4. The mutual data outage probability $\mathcal{M O P}$ versus the transmit power $P_{p}$ at PT, with different values of transmission rates $R_{p}$ and $R_{s}$, for $N_{s}=4$, $N_{p}=8, P_{s}=30 \mathrm{dBm}, E_{p}=40 \mathrm{dBm}, E_{m}=20 \mathrm{dBm}$, and $E_{0}=610^{-4}$.

rate $R_{s}$ as in Fig. 4, independently of $N_{p}$ and $R_{p}$. This result is in agreement with the asymptotic result discussed in Section V-E, when $P_{p}$ goes to zero. However, for $P_{p} \geq 30 \mathrm{dBm}, \mathcal{M O P}$ follows the performance of the primary data outage probability and decreases as we increase the number of antennas $N_{p}$ as in Fig. 3. Also, it decreases as we decrease both transmission rates $R_{p}$ and $R_{s}$ as in Fig. 4. We can see also that the AS scheme most likely outperforms the TS scheme. But, when $N_{p}=4$ and $35 \mathrm{dBm}<P_{p}<45 \mathrm{dBm}$ in Fig. 3, the TS scheme performs a slightly better than the AS scheme in terms of $\mathcal{M O P}$ in Fig. 3. Also, when $R_{p}=1$ and $30 \mathrm{dBm}<P_{p}<40$ $\mathrm{dBm}$ in Fig. 4, the TS scheme also performs a slightly better than the AS scheme in terms of $M O P$.

In Fig. 5, we have plotted the power outage $\mathcal{P O}$ and the average harvested energy $\bar{Q}$ versus the transmit power $P_{p}$ at PT, with different values of number of antennas at PR and ST and different values of transmission rates $R_{p}$ and $R_{s}$. We can always see the agreement between the analytic expressions and the Monte Carlo simulations. First, we can see that the power
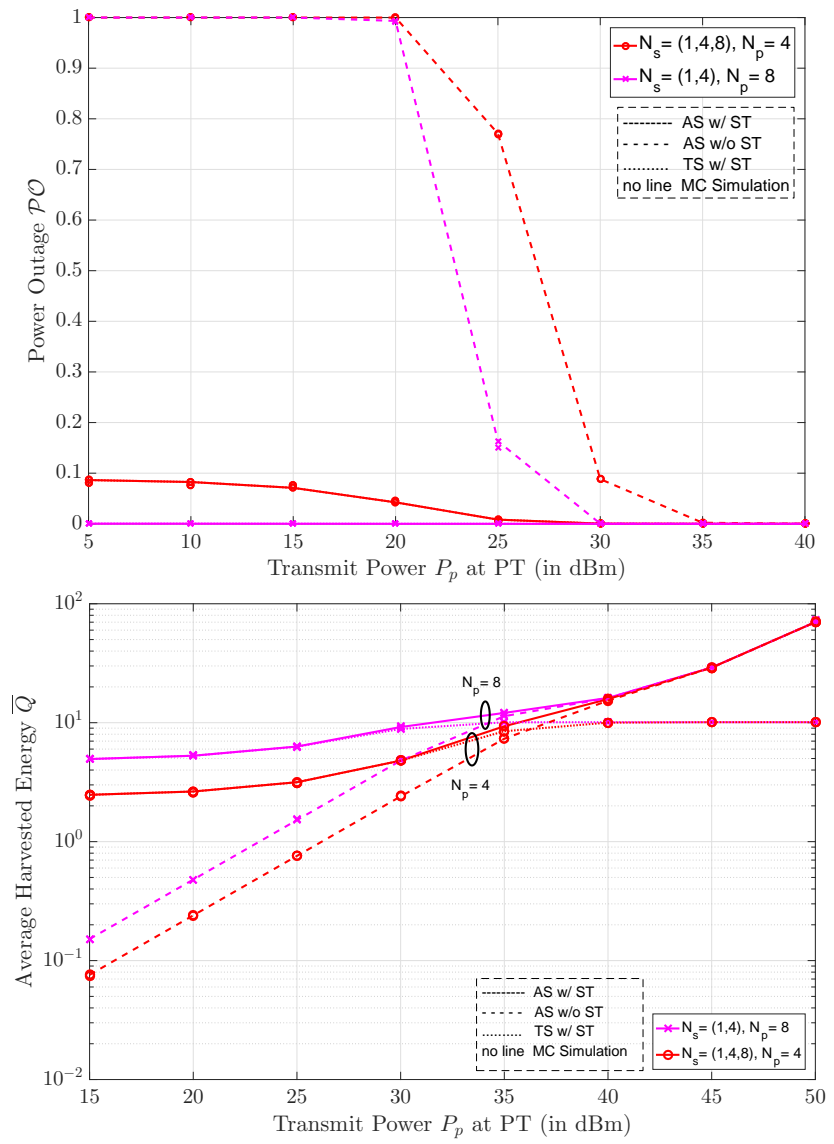

Figure 5. The power outage $\mathcal{P} O$ and the average harvested energy $\bar{Q}$ versus the transmit power $P_{p}$ at PT, with different values of number of antennas at $\mathrm{PR}$ and $\mathrm{ST}$ and different values of transmission rates $R_{p}$ and $R_{s}$, for $P_{s}=30$ $\mathrm{dBm}, \gamma_{q}=30 \mathrm{dBm}, E_{p}=40 \mathrm{dBm}, E_{m}=20 \mathrm{dBm}$, and $E_{0}=610^{-4}$.

outage decreases as we increase $P_{p}$ and $N_{p}$, independently of the number of antennas $N_{s}$. Also, we can see that the presence of ST is always beneficial in terms of the power outage at PR. Moreover, the TS and AS scheme perform alike in the presence of ST in terms of power outage. Recall that, here, we are in the case when $\gamma_{q}<E_{t}$. If not, the TS scheme will certainly perform worse as its power outage is equal to one when $\gamma_{q}$ exceeds $E_{t}$. On the other hand, we can see that the average harvested energy at PR increases as we increase $P_{p}$ and $N_{p}$, independently of $N_{s}$. The presence of ST is beneficial in terms of average harvested energy for $P_{p}<40 \mathrm{dBm}$ and the advantage of the use of the AS scheme over the TS scheme is highlighted for $P_{p}>30 \mathrm{dBm}$. Moreover, the AS scheme cannot exceed the value of $E_{t}$ which is here approximatively equal to 10. At this point, we can conclude that, in terms of data outage, the TS scheme may perform better than the AS scheme in some cases, as we have seen in Figs. 3 and 4. But, in terms of energy, the performance of the AS scheme is always better or equal to the TS scheme. So, the preference of the TS scheme over the AS scheme is restricted only to some special cases. Moreover, we have seen that the presence of ST is beneficial in terms of data and energy metrics, i.e. $\mathcal{P}_{P, \text { out }}, \mathcal{P} O$, and $\bar{Q}$. Finally, we have seen that increasing $N_{s}$ is beneficial for data metrics such as $\mathcal{P}_{S \text {,out }}$ and $\mathcal{M O P}$, while 
increasing $N_{p}$ is beneficial for both data and energy metrics, i.e. $\mathcal{P}_{P, \text { out }}, \mathcal{M O P}, \mathcal{P O}$, and $\bar{Q}$.

\section{B. Secondary Transmit Power}
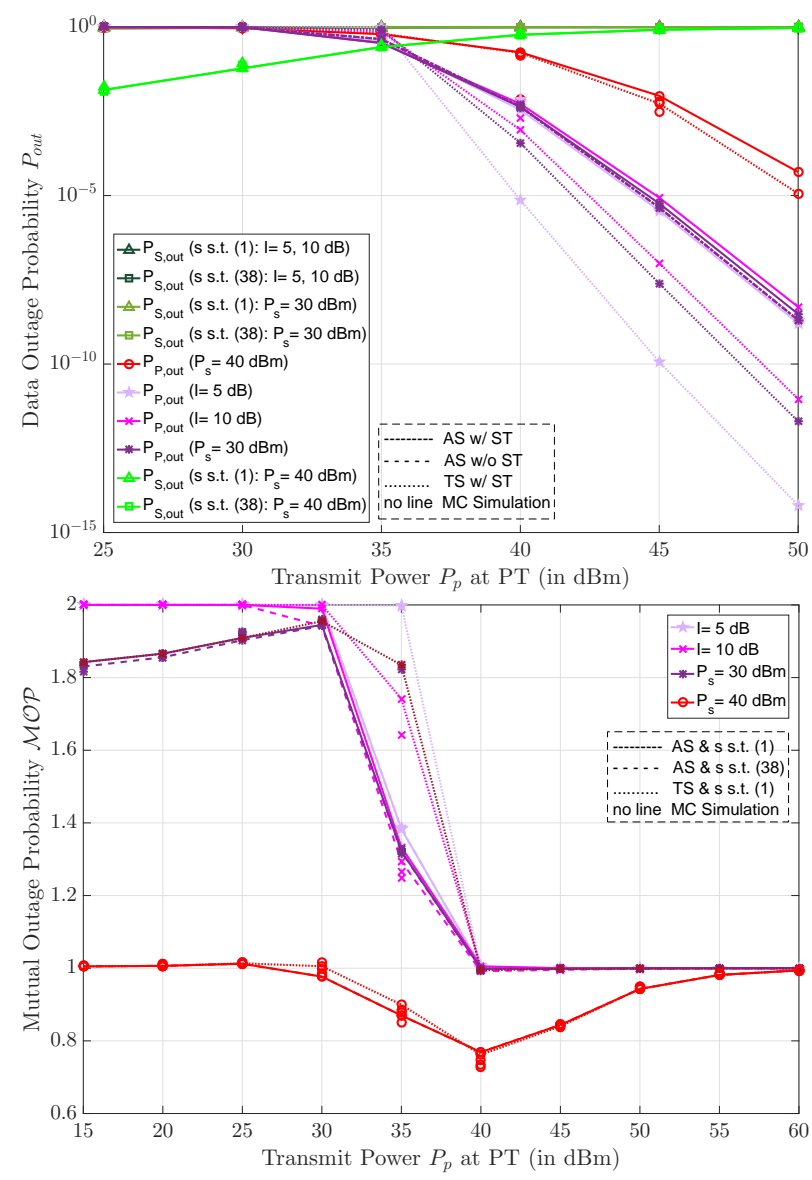

Figure 6. The primary, secondary and mutual data outage probabilities versus the transmit power $P_{p}$ at PT, with different values of transmit power $P_{s}$ at ST, for $N_{s}=4, N_{p}=8, R_{s}=R_{p}=2, E_{p}=40 \mathrm{dBm}, E_{m}=20 \mathrm{dBm}$, and $E_{0}=610^{-4}$.

In Fig. 6, we have plotted the primary, secondary and mutual data outage probabilities versus the transmit power $P_{p}$ at PT, with different values of transmit power $P_{s}$ at ST, for $N_{s}=4, N_{p}=8$, and $R_{s}=R_{p}=2$. The transmit power at ST is either equal to a fixed value (e.g. $30 \mathrm{dBm}$ and $40 \mathrm{dBm}$ ) or constrained to a maximum tolerable interference $I$ at PR based on the mean-value knowledge of CSI at ST as in [12], i.e. $P_{s}=\min \left(P_{p}, \frac{I \sigma_{p}^{2}}{N_{p} \lambda_{s p}}\right)$. We considered two values of $I$ (e.g. $5 \mathrm{~dB}$, and $10 \mathrm{~dB}$ ). First, we can see that the best performance of the secondary data outage probability is when $P_{s}=40 \mathrm{dBm}$, while the best performance of the primary data outage probability is when $P_{s} \neq 40 \mathrm{dBm}$. For $P_{p}>35 \mathrm{dBm}$, the primary data outage probability with $I=5 \mathrm{~dB}$ outperforms the other cases. We can see also that the use of the TS scheme is more preferable when the transmit power at PT becomes large while the transmit power at ST becomes low, e.g. when $P_{p}>35 \mathrm{dBm}$ and $I=5$ $\mathrm{dB}$. This observation makes sense as the use of the AS scheme won't be advantageous neither in terms of energy nor in terms of data when the ST-PR interference becomes low. Also, we can see that the presence of ST is more valuable for the primary data outage probability when $P_{s}$ decreases. In terms of the mutual data outage probability, the best performance is achieved when $P_{s}=40 \mathrm{dBm}$ and minimized when $P_{p}=P_{s}$. When $P_{s} \neq 40 \mathrm{dBm}$ and $P_{p} \leq 30 \mathrm{dBm}$, the mutual data outage probability performs better when $P_{s}=30 \mathrm{dBm}$ than the one when $I=5 \mathrm{~dB}$ and $I=10 \mathrm{~dB}$, while it performs alike for $P_{s} \neq 40 \mathrm{dBm}$ and $P_{p}>30 \mathrm{dBm}$ and converges to one when $P_{p} \geq 40 \mathrm{dBm}$. In all cases, we can see that, in terms of $\mathcal{M O P}$, the AS scheme performs better than the TS scheme and both antenna selection schemes at ST perform closely.
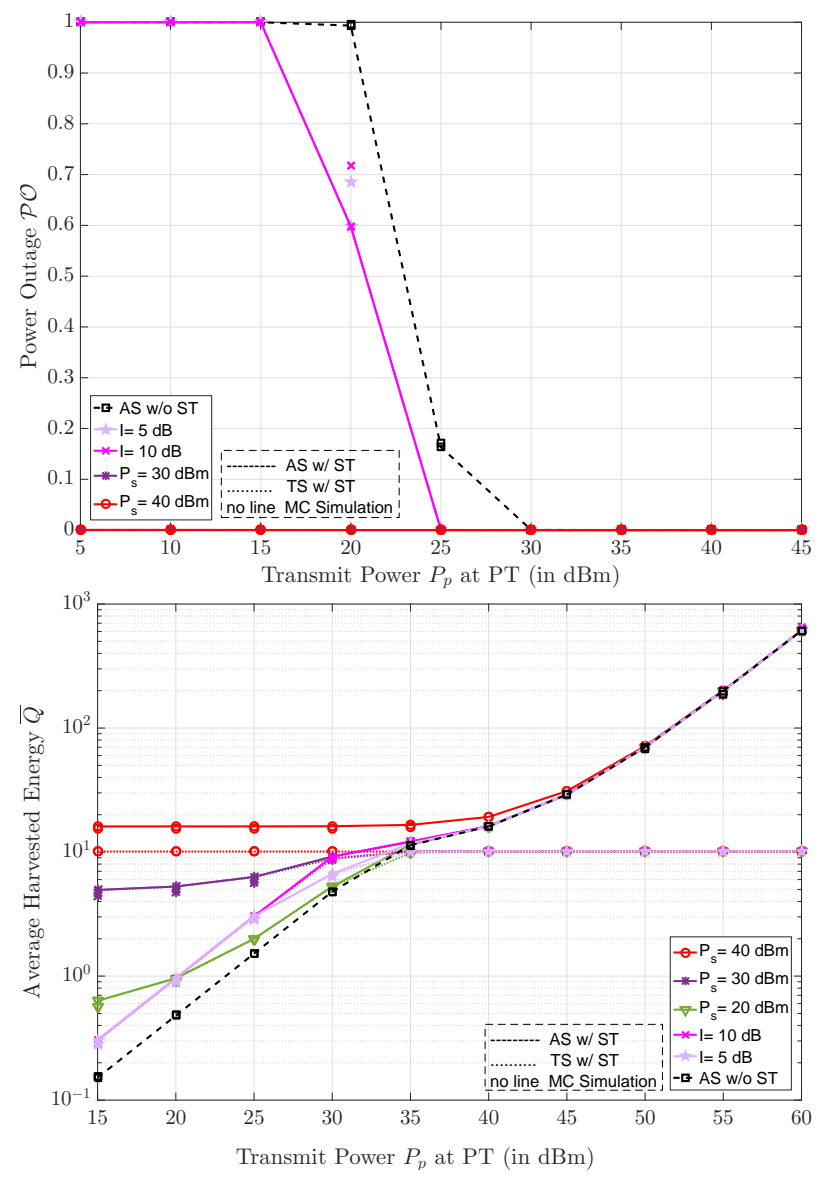

Figure 7. The power outage $\mathcal{P} O$ and the average harvested energy $\bar{Q}$ versus the transmit power $P_{p}$ at PT, with different values of transmit power $P_{s}$ at $\mathrm{ST}$, for $N_{s}=4, N_{p}=8, R_{s}=R_{p}=2, \gamma_{q}=30 \mathrm{dBm}, E_{p}=40 \mathrm{dBm}, E_{m}=20$ $\mathrm{dBm}$, and $E_{0}=610^{-4}$.

In Fig. 7, we have plotted the power outage $\mathcal{P} O$ and the average harvested energy $\bar{Q}$ versus the transmit power $P_{p}$ at PT, with different values of transmit power $P_{s}$ at ST, for $N_{s}=4, N_{p}=8, R_{s}=R_{p}=2$, and $\gamma_{q}=30 \mathrm{dBm}$. First, we can see that the power outage improves as we increase $P_{s}$. The presence of ST is advantageous to PR in terms of power outage. Also, the TS and AS schemes perform alike. When $I=5 \mathrm{~dB}$ and $I=10 \mathrm{~dB}$, the performance of the power outage is the same for $P_{p} \leq 25 \mathrm{dBm}$. Also, using an interference constraint at PR worsens power outage for $P_{p} \leq 25 \mathrm{dBm}$. For higher $P_{p}$, the performance in terms of the power outage is alike for all cases. In terms of average harvested energy, the best performance is achieved when $P_{s}=40 \mathrm{dBm}$. The 
average harvested energy with $I=10 \mathrm{~dB}$ outperforms the one with $I=5 \mathrm{~dB}$, for $P_{p}>25 \mathrm{dBm}$, and performs alike the one with $P_{s}=30 \mathrm{dBm}$, for $P_{p} \geq 30 \mathrm{dBm}$. Also, we can observe the advantage due to the presence of ST and the advantage due to the use of AS scheme over the TS scheme. Finally, we can conclude that increasing the transmit power at ST improves the data metrics such as $\mathcal{P}_{S, \text { out }}$ and $\mathcal{M O P}$ and the energy metrics $\mathcal{P} O$ and $\bar{Q}$.

\section{ST-PR Interference Channel Variance $\lambda_{s p}$ and Energy Threshold $E_{t}$}
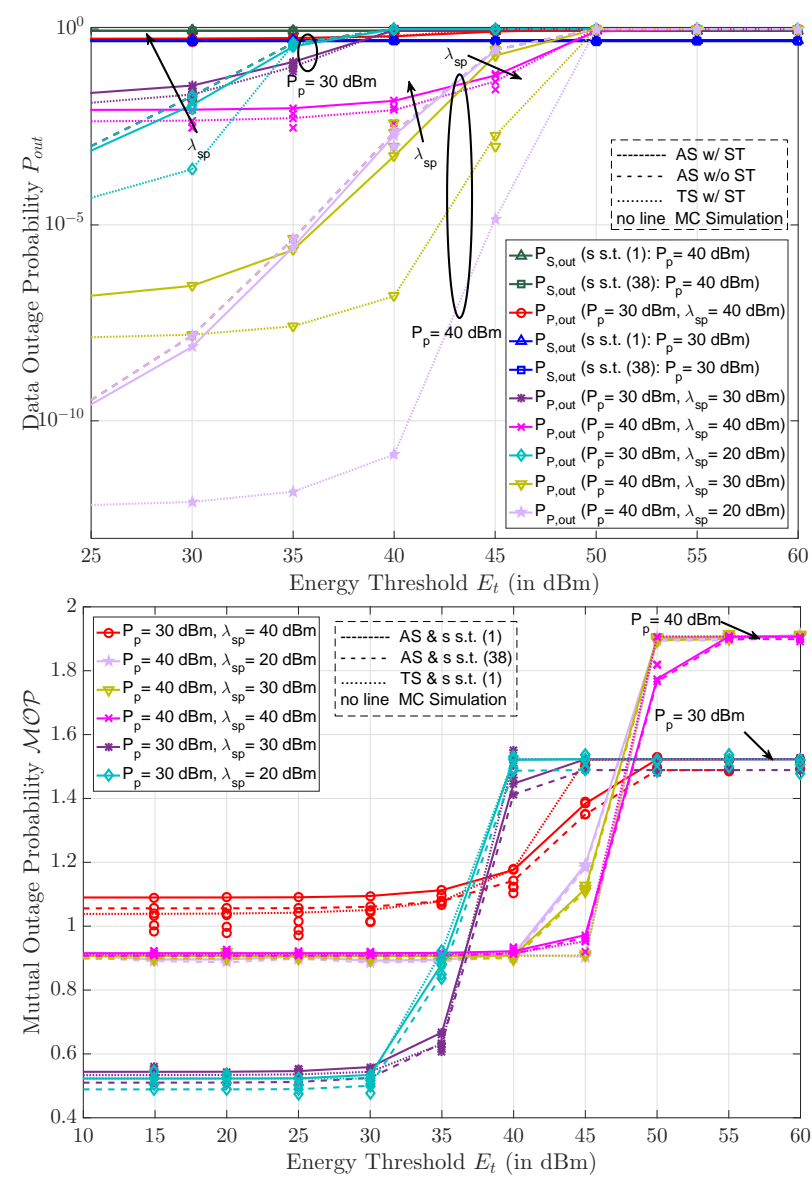

Figure 8 . The primary, secondary and mutual data outage probabilities versus the energy threshold $E_{t}$, with different values of transmit power $P_{p}$ at PT and the interference channel variance $\lambda_{s p}$, for $N_{s}=4, N_{p}=8, R_{s}=R_{p}=1$, and $P_{s}=30 \mathrm{dBm}$.

In Fig. 8, we have plotted the primary, secondary and mutual data outage probabilities versus the energy threshold $E_{t}$, with different values of transmit power $P_{p}$ at PT and the interference channel variance $\lambda_{s p}$, for $N_{s}=4, N_{p}=8$, $R_{s}=R_{p}=1$, and $P_{s}=30 \mathrm{dBm}$. First of all, we can see that the presence of ST improves $\mathcal{P}_{P, \text { out }}$ for any $E_{t}$ and the AS scheme almost performs better that the TS scheme in terms $\mathcal{M O P}$. The TS scheme outperforms the AS scheme in terms of $\mathcal{P}_{P, \text { out }}$ for low $E_{t}$. We can see also that the primary data outage probability increases as $E_{t}$ increases. In fact, as $E_{t}$ increases, the energy requirements at PR increase and more antennas are needed to harvest the energy while few antennas are left to decode the information data. This results in increasing the primary data outage probability. Moreover, the secondary data outage probability remains constant. This result is expected since the EH constraint applies only to the PR side. In addition, as we increase $P_{p}$, the primary data outage probability increases and the secondary data outage probability decreases, as in Figs. 2 and 6. Moreover, if we increase the ST-PR interference channel variance $\lambda_{s p}$, the secondary data outage probability remains constant. But, the behavior of the primary data outage probability in function of $\lambda_{s p}$ depends on the value $E_{t}$. In fact, it increases for low values of $E_{t}$ and decreases for high values of $E_{t}$, as $\lambda_{s p}$ increases. For example, when $P_{p}=40 \mathrm{dBm}$, the primary data outage probability performs better with $\lambda_{s p}=20 \mathrm{dBm}$ for $E_{t} \leq 35 \mathrm{dBm}$, then performs better with $\lambda_{s p}=30 \mathrm{dBm}$ for $35 \leq E_{t}<45 \mathrm{dBm}$, and finally performs better with the highest $\lambda_{s p}=40 \mathrm{dBm}$. This behavior of $\mathcal{P}_{P, \text { out }}$ can be explained by the fact that for low $E_{t}$, the PR antennas are more used to decode the information data and the ST-PR interference is harming the data decoding task. But, for high $E_{t}$, the PR antennas are more used to harvest the energy and the ST-PR interference is desired to get more energy. Furthermore, the mutual data outage probability is constant for $E_{t} \leq 30 \mathrm{dBm}$ and for $E_{t}>50 \mathrm{dBm}$ and increases when $E_{t}$ is in-between. The variation of $\mathcal{M O P}$ in function of $P_{p}$ and $\lambda_{s p}$ depends on the range of $E_{t}$. For high $E_{t}$, $\mathcal{M O P}$ is constant with respect to $\lambda_{s p}$ and decreases when we decrease $P_{p}$. This constant behavior is expected as explained in Section V-E. In fact, when $E_{t} \rightarrow \infty$, the primary data outage probability is equal to 1 and the performance of $\mathcal{M O P}$ is related to the performance of $\mathcal{P}_{S, \text { out }}$ which only depends on $P_{p}$ and increases as we increase $P_{p}$. For $35 \mathrm{dBm}<E_{t}<50$ $\mathrm{dBm}, \mathcal{M O P}$ decreases as we increase both $P_{p}$ and $\lambda_{s p}$. But, for low $E_{t}$, the constant behavior of $\mathcal{M O P}$ is expected in Section $\mathrm{V}$-E when $E_{t} \rightarrow 0$. The dependence of $\mathcal{M O P}$ in function of $P_{p}$ and $\lambda_{s p}$ is variant. $\mathcal{M O P}$ decreases as we decrease both $P_{p}$ and $\lambda_{s p}$, expect when $P_{p}=30 \mathrm{dBm}$ and $\lambda_{s p}=40 \mathrm{dBm}$.

In Fig. 9, we have plotted the power outage $\mathcal{P O}$ and the average harvested energy $\bar{Q}$ versus the energy threshold $E_{t}$, with different values of transmit power $P_{p}$ at PT and the interference channel variance $\lambda_{s p}$, for $N_{s}=4, N_{p}=8$, $R_{s}=R_{p}=1, P_{s}=30 \mathrm{dBm}$ and $\gamma_{q}=30 \mathrm{dBm}$. First, the power outage decreases as we increase $E_{t}, P_{p}$ and $\lambda_{s p}$. Moreover, the average harvested energy increases as we increase $E_{t}, P_{p}$ and $\lambda_{s p}$. So, we can summarize that increasing the energy threshold improves the energy metrics while it harms the data metrics. Moreover, increasing the ST-PR interference channel variance $\lambda_{s p}$ improves the energy metrics while its dependence in terms of data metrics depends on the value of $E_{t}$.

\section{Conclusion}

In this paper, we have considered the performance analysis of the simultaneous wireless information and power transfer for the SS in the CR network with a multi-antenna PR that has EH capabilities using the AS technique. We have studied the incentive of the SS by evaluating the exact expressions of the energy metrics which are the average harvested energy and the power outage at PR and the data metrics which are 

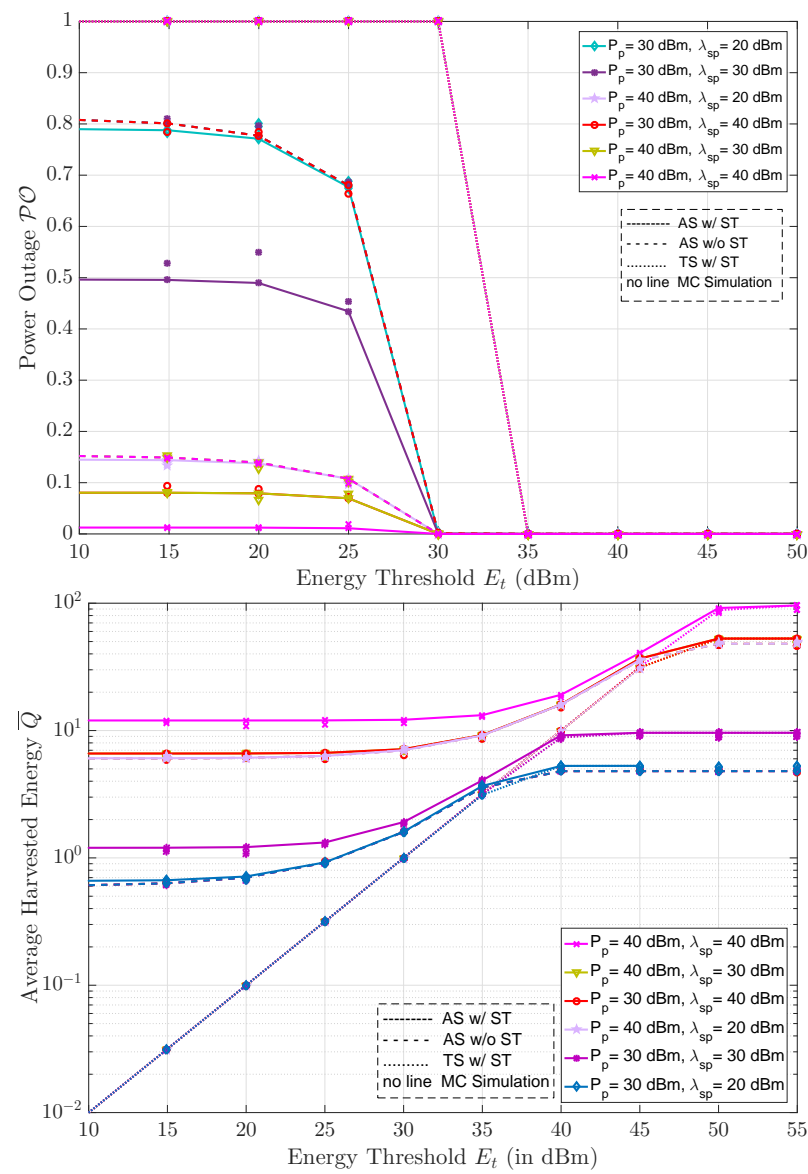

Figure 9. The power outage $\mathcal{P} O$ and the average harvested energy $\bar{Q}$ versus the energy threshold $E_{t}$, with different values of transmit power $P_{p}$ at PT and the interference channel variance $\lambda_{s p}$, for $N_{s}=4, N_{p}=8, R_{s}=R_{p}=1$, $P_{s}=30 \mathrm{dBm}$ and $\gamma_{q}=30 \mathrm{dBm}$.

the primary and secondary data outage probability and the mutual outage probability (MOP). We have presented some special cases and asymptotic results of the analytic results. We have validated our analytic expressions by comparing them to the Monte Carlo simulation, and we have shown the benefits due to the presence of ST through the simulation results in terms of data and energy metrics. We have compared our analytic results to the TS scheme, and we have shown that the AS scheme performs better that the TS scheme in terms of the data metric $\mathcal{M O P}$ and the energy metrics $\mathcal{P} O$, and $\bar{Q}$. The TS scheme outperforms the AS scheme only for some specific cases in terms of $\mathcal{P}_{P, \text { out }}$. Moreover, we have shown the impact of increasing the number of antennas at ST and $\mathrm{PR}$, the transmit power at PT and ST, the ST-PR interference channel variance, the energy threshold, and the primary and secondary transmission rates on all the analytic results of the data and energy performance metrics.

\section{APPENDIX A}

Proof of Lemma 1

Recall, $s$ is selected such as

$$
s=\underset{1 \leq l \leq N_{s}}{\arg \max } \frac{\left|h_{l d}\right|^{2}}{\sum_{j=1}^{N_{p}}\left|h_{l j}\right|^{2}} .
$$

If we denote $X_{l}=\left|h_{l d}\right|^{2}, Y_{l}=\sum_{j=1}^{N_{p}}\left|h_{l j}\right|^{2}$ and $Z_{l}=\frac{X_{l}}{Y_{l}}$, the PDF of $\left|h_{s d}\right|^{2}$ can be written as

$$
f_{\left|h_{s d}\right|^{2}}(x)=\int_{0}^{\infty} f_{X_{l} \mid Z_{l}=z}(x \mid z) f_{\max \left(Z_{l}\right)}(z) \mathrm{d} z
$$

and, correspondingly, the PDF of $\gamma_{s d}$ is written as

$$
f_{\gamma_{s d}}(x)=\frac{1}{\overline{\gamma_{s_{1}}}} f_{\left|h_{s d}\right|^{2}}\left(\frac{x}{\overline{\gamma_{s_{1}}}}\right) \text {. }
$$

Thus, we need to derive the conditional $\mathrm{CDF}$ of $X_{l}$ given $Z_{l}=z$ and the PDF of the maximum of $Z_{l}$ in order to obtain the PDFs of $\left|h_{s d}\right|^{2}$ and $\gamma_{s d}$.

\section{A. Derivation of $f_{X_{l} \mid Z_{l}=z}(x \mid z)$}

First, we express the conditional PDF of $X_{l}$ given $Z_{l}=z$ as the ratio between the joint PDF of $X_{l}$ and $Z_{l}$ and the marginal of $Z_{l}$

$$
f_{X_{l} \mid Z_{l}=z}(x \mid z)=\frac{f_{X_{l}, Z_{l}}(x, z)}{f_{Z_{l}}(z)},
$$

where $f_{X_{l}, Z_{l}}(x, z)$ and $f_{Z_{l}}(z)$ can be derived from their CDFs:

$$
\begin{aligned}
f_{X_{l}, Z_{l}}(x, z) & =\frac{\delta^{2} \mathcal{F}_{X_{l}, Z_{l}}(x, z)}{\delta x \delta z}, \\
f_{Z_{l}}(z) & =\frac{\delta \mathcal{F}_{Z_{l}}(z)}{\delta z} .
\end{aligned}
$$

First, we show that the joint $\mathrm{CDF}$ of $X_{l}$ and $Z_{l}$ can be written as the integral of the product of the CDF of $X_{l}$ and the PDF of $Y_{l}$ as

$$
\begin{aligned}
\mathcal{F}_{X_{l}, Z_{l}}(x, z) & =\int_{0}^{\infty} \mathcal{F}_{X_{l}}(\min (x, y z)) f_{Y_{l}}(y) \mathrm{d} y \\
& =1-\frac{1}{\Gamma\left(N_{p}\right)} \frac{1}{\lambda_{s p}^{N_{p}}}\left(\frac{z}{\lambda_{s d}}+\frac{1}{\lambda_{s p}}\right)^{-N_{p}} \gamma\left(N_{p}, \frac{x}{z}\left(\frac{z}{\lambda_{s d}}+\frac{1}{\lambda_{s p}}\right)\right) \\
& -e^{-\frac{x}{\lambda_{s d}}} \frac{1}{\Gamma\left(N_{p}\right)} \Gamma\left(N_{p}, \frac{1}{\lambda_{s p}} \frac{x}{z}\right)
\end{aligned}
$$

(72) was obtained after substituting the CDF of $X_{l}$ and the PDF of $Y_{l}$, and using the integral presentations of the lower and upper incomplete Gamma functions in [28, (3.381.1)] and [28, (3.381.3)], respectively. After deriving $\mathcal{F}_{X_{l}, Z_{l}}(x, z)$ with respect to $x$ and $z$, we deduce $f_{X_{l}, Z_{l}}(x, z)$ as

$$
f_{X_{l}, Z_{l}}(x, z)=\frac{1}{\Gamma\left(N_{p}\right)} \frac{1}{\lambda_{s d} \lambda_{s p}^{N_{p}}} \frac{x^{N_{p}}}{z^{N_{p}+1}} e^{-x\left(\frac{1}{\lambda_{s d}}+\frac{1}{\lambda_{s p}} \frac{1}{z}\right)} .
$$

In addition, in order to derive the PDF of $Z_{l}$, we first investigate its $\mathrm{CDF}$ and we show that it can be written as function of the CDF of $X_{l}$ and the PDF of $Y_{l}$ as

$$
\mathcal{F}_{Z_{l}}(z)=\int_{0}^{\infty} \mathcal{P}\left(X_{l} \leq y z\right) f_{Y_{l}}(y) \mathrm{d} y=\int_{0}^{\infty}\left(\int_{0}^{z} y f_{X_{l}}(t y) \mathrm{d} t\right) f_{Y_{l}}(y) \mathrm{d} y .
$$

Deriving the CDF of $Z_{l}$ in (74) with respect to $z$, we obtain the expression of the PDF of $Z_{l}$ as

$$
f_{Z_{l}}(z)=\int_{0}^{\infty} y f_{X_{l}}(z y) f_{Y_{l}}(y) d y=\frac{N_{p} \lambda_{s d}^{N_{p}} \lambda_{s p}}{\left(z \lambda_{s p}+\lambda_{s d}\right)^{N_{p}+1}} .
$$

Substituting (73) and (75) in (68), we obtain the conditional PDF $f_{X_{l} \mid Z_{l}=z}(x \mid z)$ as

$$
f_{X_{l} \mid Z_{l}=z}(x \mid z)=\frac{x^{N_{p}}}{\Gamma\left(N_{p}+1\right)}\left(\frac{1}{\lambda_{s d}}+\frac{1}{z \lambda_{s p}}\right)^{N_{p}+1} e^{-x\left(\frac{1}{\lambda_{s d}}+\frac{1}{\lambda_{s p}} \frac{1}{z}\right)} .
$$




\section{B. Derivation of $f_{\max \left(Z_{l}\right)}(z)$}

On the other hand, we express the CDF of the maximum of $Z_{l}$ in order to obtain later its PDF. We show that the CDF of the maximum of $Z_{l}$ is equal to the product of $N_{s}$ CDFs of $Z_{l}$ as

$$
\mathcal{F}_{\max \left(Z_{l}\right)}(z)=\mathcal{P}\left(\max \left(Z_{l}\right) \leq z\right)=\left(\mathcal{F}_{Z_{l}}(z)\right)^{N_{s}},
$$

where $\mathcal{F}_{Z_{l}}(z)$ can be derived from its PDF given in (75) as

$$
\mathcal{F}_{Z_{l}}(z)=\int_{0}^{z} f_{Z_{l}}(z) \mathrm{d} z=1-\frac{\lambda_{s d}^{N_{p}}}{\left(z \lambda_{s p}+\lambda_{s d}\right)^{N_{p}}} .
$$

Finding the derivative of (77) with respect to $z$, we express the PDF of the maximum of $Z_{l}$ as function of the PDF and $\mathrm{CDF}$ of $Z_{l}$, then we substitute (75) and (78) in the derivative of (77) to obtain

$$
\begin{aligned}
& f_{\max \left(Z_{l}\right)}(z)=N_{s} f_{Z_{l}}(z)\left(\mathcal{F}_{Z_{l}}(z)\right)^{N_{s}-1} \\
& =\frac{N_{s} N_{p} \lambda_{s d}^{N_{p}} \lambda_{s p}}{\left(z \lambda_{s p}+\lambda_{s d}\right)^{N_{p}+1}}\left(1-\frac{\lambda_{s d}^{N_{p}}}{\left(z \lambda_{s p}+\lambda_{s d}\right)^{N_{p}}}\right)^{N_{s}-1} \\
& =\frac{N_{s} N_{p} \lambda_{s d}^{N_{p}} \lambda_{s p}}{\left(z \lambda_{s p}+\lambda_{s d}\right)^{N_{p}+1}} \sum_{j=0}^{N_{s}-1}\left(\begin{array}{c}
N_{s}-1 \\
j
\end{array}\right)\left(-\frac{\lambda_{s d}^{N_{p}}}{\left(z \lambda_{s p}+\lambda_{s d}\right)^{N_{p}}}\right)^{j},
\end{aligned}
$$

where (80) was obtained from (79) using the Binomial expansion [28].

\section{PDF of $\gamma_{s d}$}

Then, we substitute (76) and (80) in (66) to obtain the PDF of $\gamma_{s d}$ as

$$
\begin{aligned}
f_{\left|h_{s d \mid}\right|^{2}}(x) & =\int_{0}^{\infty} f_{X_{l} \mid Z_{l}=z}(x \mid z) f_{\max \left(Z_{l}\right)}(z) \mathrm{d} z \\
& =\frac{N_{s}}{\lambda_{s d}} e^{-\frac{x}{\lambda_{s d}}}+\frac{N_{s}}{\Gamma\left(N_{p}\right)} \frac{x^{N_{p}}}{\lambda_{s d} \lambda_{s p}^{N_{p}}} \sum_{j=1}^{N_{s}-1}\left(\begin{array}{c}
N_{s}-1 \\
j
\end{array}\right) \\
& \times(-1)^{j} \lambda_{s d}^{N_{p} j} \int_{0}^{\infty} \frac{e^{-x\left(\frac{1}{\lambda_{s d}}+\frac{1}{\lambda_{s p}} \frac{1}{z}\right)}}{z^{N_{p}+1}\left(z \lambda_{s p}+\lambda_{s d}\right)^{N_{p} j}} \mathrm{~d} z .
\end{aligned}
$$

Using the change of variables as $v=\lambda_{s d}\left(\frac{1}{\lambda_{s d}}+\frac{1}{\lambda_{s p}} \frac{1}{z}\right)$, we show that (82) can be written as

$$
\begin{aligned}
f_{\left|h_{s d \mid}\right|^{2}}(x) & =\frac{N_{s}}{\lambda_{s d}} e^{-\frac{x}{\lambda_{s d}}}+\frac{N_{s}}{\Gamma\left(N_{p}\right)} \frac{x^{N_{p}}}{\lambda_{s d}^{N_{p}+1}} \sum_{j=1}^{N_{s}-1}\left(\begin{array}{c}
N_{s}-1 \\
j
\end{array}\right)(-1)^{j} \\
& \times \int_{1}^{\infty} v^{-N_{p} j}(v-1)^{N_{p}(j+1)-1} e^{-\frac{x v}{\lambda_{s d}}} \mathrm{~d} v \\
& =\frac{N_{s}}{\lambda_{s d}} e^{-\frac{x}{\lambda_{s d}}}+\frac{N_{s}}{\Gamma\left(N_{p}\right) \lambda_{s d}}\left(\frac{x}{\lambda_{s d}}\right)^{\frac{N_{p}-1}{2}} e^{-\frac{x}{2 \lambda_{s d}}} \sum_{j=1}^{N_{s}-1}\left(\begin{array}{c}
N_{s}-1 \\
j
\end{array}\right) \\
& \times(-1)^{j} \Gamma\left(N_{p}(j+1)\right) W_{\frac{-N_{p}(2 j+1)+1}{2}, \frac{-N_{p}}{2}}\left(\frac{x}{\lambda_{s d}}\right)
\end{aligned}
$$

where (84) was obtained using $[28,(3.384 .4)]$ and $W_{\lambda, \mu}(\cdot)$ is the Whittaker function [28]. Or, equivalently, we can compute the PDF of $f_{\left|h_{s d}\right|^{2}}(x)$ in a different way by applying the Binomial expansion to (83). Then, we use the equality in $[28,(3.382 .4)]$ to express the PDF of $f_{\left|h_{s d}\right|^{2}}(x)$ using the upper incomplete Gamma function as

$$
\begin{aligned}
f_{\left|h_{s d}\right|^{2}}(x) & =\frac{N_{s}}{\lambda_{s d}} e^{-\frac{x}{\lambda_{s d}}}+\frac{N_{s}}{\Gamma\left(N_{p}\right) \lambda_{s d}} \sum_{j=1}^{N_{s}-1} \sum_{i=0}^{N_{p}(j+1)-1}\left(\begin{array}{c}
N_{s}-1 \\
j
\end{array}\right) \\
& \times\left(\begin{array}{c}
N_{p}(j+1)-1 \\
i
\end{array}\right)(-1)^{j+i}\left(\frac{x}{\lambda_{s d}}\right)^{i} \Gamma\left(N_{p}-i, \frac{x}{\lambda_{s d}}\right) .
\end{aligned}
$$

Subsequently, the PDF of $\gamma_{s d}$ is given by

$$
\begin{aligned}
f_{\gamma_{s d}}(x) & =\frac{N_{s}}{\alpha_{1}} e^{-\frac{x}{\alpha_{1}}}+\frac{N_{s}}{\Gamma\left(N_{p}\right) \alpha_{1}} \sum_{j=1}^{N_{s}-1} \sum_{i=0}^{N_{p}(j+1)-1}\left(\begin{array}{c}
N_{s}-1 \\
j
\end{array}\right) \\
& \times\left(\begin{array}{c}
N_{p}(j+1)-1 \\
i
\end{array}\right)(-1)^{j+i}\left(\frac{x}{\alpha_{1}}\right)^{i} \Gamma\left(N_{p}-i, \frac{x}{\alpha_{1}}\right) .
\end{aligned}
$$

\section{D. $C D F$ of $\gamma_{s d}$}

By using the integration by parts in (82), the CDF of $\gamma_{s d}$ is given by

$$
\begin{aligned}
\mathcal{F}_{\left|h_{s d \mid}\right|^{2}}(y) & =N_{s}\left(1-e^{-\frac{y}{\lambda_{s d}}}\right)+N_{s} \sum_{j=1}^{N_{s}-1}\left(\begin{array}{c}
N_{s}-1 \\
j
\end{array}\right)(-1)^{j}\left[\frac{1}{j+1}\right. \\
& -\frac{\Gamma\left(N_{p}(j+1)\right)}{\Gamma\left(N_{p}\right)}\left(\frac{y}{\lambda_{s d}}\right)^{\frac{N_{p}}{2}} e^{\left.-\frac{y}{2 \lambda_{s d}} W_{\frac{-N_{p}(2 j+1)}{2}}, \frac{-N_{p}-1}{2}\left(\frac{y}{\lambda_{s d}}\right)\right],}
\end{aligned}
$$

where (87) was obtained using [28, (3.471.7)]. Or, equivalently, using (86), the CDF of $\gamma_{s d}$ is written as

$$
\begin{aligned}
\mathcal{F}_{\gamma_{s d}}(y) & =N_{s}\left(1-e^{-\frac{y}{\alpha_{1}}}\right)+\frac{N_{s}}{\Gamma\left(N_{p}\right) \alpha_{1}} \sum_{j=1}^{N_{s}-1} \sum_{i=0}^{N_{p}(j+1)-1}\left(\begin{array}{c}
N_{s}-1 \\
j
\end{array}\right) \\
& \times\left(\begin{array}{c}
N_{p}(j+1)-1 \\
i
\end{array}\right) \frac{(-1)^{j+i}}{i+1}\left[\gamma\left(N_{p}+1, \frac{y}{\alpha_{1}}\right)\right. \\
& \left.+\alpha_{1}\left(\frac{y}{\alpha_{1}}\right)^{i+1} \Gamma\left(N_{p}-i, \frac{y}{\alpha_{1}}\right)\right],
\end{aligned}
$$

which was obtained by expressing the incomplete Gamma function in (86) by its integral presentation as in [28, (3.381.3)], permuting the double integrals and solving the inner integral using $[28,(3.381 .1)]$.

\section{APPENDIX B}

Proof of Lemma 2

We recall that $Q(k)$, for $k=0, \ldots, N_{p}-1$ is given by

$$
Q(k)=\zeta \sum_{j=k+1}^{N_{p}}\left(P_{p}\left|h_{p j}\right|^{2}+P_{s}\left|h_{s j}\right|^{2}\right)=X+Y
$$

with $X=\zeta P_{p} \sum_{j=k+1}^{N_{p}}\left|h_{p j}\right|^{2}$, and $Y=\zeta P_{s} \sum_{j=k+1}^{N_{p}}\left|h_{s j}\right|^{2}$. The PDFs of $X$ and $Y$ are given by

$$
\begin{aligned}
f_{X}(x) & =\frac{1}{\Gamma\left(N_{p}-k\right)} \frac{x^{N_{p}-k-1}}{\left(\zeta \varepsilon_{p}\right)^{N_{p}-k}} e^{-\frac{x}{\zeta \varepsilon_{p}}}, \\
f_{Y}(y) & =\frac{1}{\Gamma\left(N_{p}-k\right)} \frac{y^{N_{p}-k-1}}{\left(\zeta \varepsilon_{s}\right)^{N_{p}-k}} e^{-\frac{y}{\zeta \varepsilon_{s}}},
\end{aligned}
$$

respectively. 


\section{A. $P D F$ of $Q(k)$}

The PDF of $Q(k)$ can be written as function of the PDFs of $X$ and $Y$ as follows:

$$
\begin{aligned}
& f^{\left(N_{p}-k\right)}(z)=\int_{0}^{z} f_{X}(t) f_{Y}(z-t) \mathrm{d} t \\
& =\frac{e^{-\frac{z}{\zeta s_{s}}}}{\left(\Gamma\left(N_{p}-k\right)\right)^{2}\left(\zeta^{2} \varepsilon_{p} \varepsilon_{s}\right)^{N_{p}-k}} \int_{0}^{z} t^{N_{p}-k-1}(z-t)^{N_{p}-k-1} e^{\frac{\varepsilon p s}{\zeta \varepsilon_{p} \varepsilon_{s}} t} \mathrm{~d} t
\end{aligned}
$$

whose resolution depends on the value $\varepsilon_{p s}$. Indeed,

- If $\varepsilon_{p s}=0$, we use [28, (3.191.1)] to show that:

$$
f^{\left(N_{p}-k\right)}(z)=\frac{1}{\Gamma\left(2\left(N_{p}-k\right)\right)} \frac{z^{2\left(N_{p}-k\right)-1}}{\left(\zeta \varepsilon_{s}\right)^{2\left(N_{p}-k\right)}} e^{-\frac{z}{\zeta \varepsilon_{s}}} .
$$

- However, if $\varepsilon_{p s} \neq 0$, we use [28, (3.383.2)] to show that:

$$
f^{\left(N_{p}-k\right)}(z)=\frac{\sqrt{\pi} z^{N_{p}-k-\frac{1}{2}} e^{-\left(\frac{1}{\varepsilon_{s}}+\frac{1}{\varepsilon_{p}}\right) \frac{z}{2 \zeta}} I_{N_{p}-k-\frac{1}{2}}\left(\frac{\varepsilon_{p s}}{\varepsilon_{p} \varepsilon_{s}} \frac{z}{2 \zeta}\right)}{\Gamma\left(N_{p}-k\right) \sqrt{\zeta^{2} \varepsilon_{p} \varepsilon_{s}}\left(\zeta \varepsilon_{p s}\right)^{N_{p}-k-\frac{1}{2}}} .
$$

Note here that, if $k=N_{p}-1$, the PDF of $Q(k)$ simplifies to

$$
f^{(1)}(z)= \begin{cases}\left.\frac{z e^{-\frac{z}{\zeta s}}}{\left(\zeta \varepsilon_{\xi}^{2}\right.}\right)^{2} & \text { if } \varepsilon_{p s}=0 \\ \frac{e^{-\frac{\varepsilon_{p}}{\zeta \varepsilon_{p}}}-e^{-\frac{z}{\zeta \varepsilon_{s}}}}{\zeta \varepsilon_{p s}}, & \text { if } \varepsilon_{p s} \neq 0\end{cases}
$$

which can be easily obtained using (93) for $k=N_{p}-1$.

\section{B. $C D F$ of $Q(k)$}

Similarly, the CDF of $Q(k)$ can be written as

$$
\begin{aligned}
& \mathcal{F}^{\left(N_{p}-k\right)}(t)=\int_{0}^{t} f_{X}(x) \mathcal{F}_{Y}(t-x) \mathrm{d} x \\
& =\frac{1}{\Gamma\left(N_{p}-k\right)^{2}\left(\zeta \varepsilon_{p}\right)^{N_{p}-k}} \int_{0}^{t} x^{N_{p}-k-1} e^{-\frac{x}{\zeta \varepsilon_{p}}} \gamma\left(N_{p}-k, \frac{t-x}{\zeta \varepsilon_{s}}\right) \mathrm{d} x .
\end{aligned}
$$

We substitute the lower incomplete Gamma function by its sum representation using $[28,(8.352 .1)]$ and we solve depending on the value of $\varepsilon_{p s}$. In fact,

- If $\varepsilon_{p s}=0$, we use the integral presentation of the lower incomplete Gamma function as in [28, (3.381.1)] and the integral equality in $[28,(3.191 .1)]$ and we show after mathematical manipulations that the $\operatorname{CDF}$ of $Q(k)$ is given by:

$$
\mathcal{F}^{\left(N_{p}-k\right)}(t)=\frac{\gamma\left(2\left(N_{p}-k\right), \frac{t}{\zeta \varepsilon_{s}}\right)}{\Gamma\left(2\left(N_{p}-k\right)\right)} .
$$

- However, if $\varepsilon_{p s} \neq 0$, we use [28, (3.381.1)] and the integral equality in $[28,(3.383 .1)]$ to show that:

$$
\begin{aligned}
\mathcal{F}^{\left(N_{p}-k\right)}(t) & =\frac{\gamma\left(N_{p}-k, \frac{t}{\zeta \varepsilon_{p}}\right)}{\Gamma\left(N_{p}-k\right)}-\left(\frac{t}{\zeta \varepsilon_{p}}\right)^{N_{p}-k} e^{-\frac{t}{\zeta \varepsilon_{s}}} \\
& \times \sum_{j=0}^{N_{p}-k-1} \frac{\left(\frac{t}{\zeta \varepsilon_{s}}\right)^{j}{ }_{1} F_{1}\left(N_{p}-k ; N_{p}-k+j+1 ; \frac{\varepsilon_{p s}}{\varepsilon_{-} s \varepsilon_{p}} \frac{t}{\zeta}\right)}{\Gamma\left(N_{p}-k+j+1\right)} .
\end{aligned}
$$

Note that, if $k=N_{p}-1$, the $\mathrm{CDF}$ of $Q\left(N_{p}-1\right)$ simplifies to

$$
\mathcal{F}^{(1)}(t)= \begin{cases}1-\left(1+\frac{t}{\zeta \varepsilon_{s}}\right) e^{-\frac{t}{\zeta s_{s}}}, & \text { if } \varepsilon_{p s}=0, \\ 1-\frac{\varepsilon_{p} e^{-\frac{t}{\varepsilon_{p}}}-\varepsilon_{s} e^{-\frac{t}{\zeta \varepsilon_{s}}}}{\varepsilon_{p s}}, & \text { if } \varepsilon_{p s} \neq 0 .\end{cases}
$$

\section{ApPendix C}

\section{Proof of Theorem 1}

Based on the EH model explained in Section III-A, the PMF of $K$ can be written, for a given $E_{t}>0$, as

$\mathcal{P}_{K}(k)= \begin{cases}0, & \text { if } k=N_{p} \\ \mathcal{P}\left(E_{t} \leq Q\left(N_{p}-1\right)\right), & \text { if } k=N_{p}-1, \\ \mathcal{P}\left(Q(k+1)<E_{t} \leq Q(k)\right), & \text { if } 1 \leq k \leq N_{p}-2, \\ \mathcal{P}\left(Q(1)<E_{t}\right), & \text { if } k=0,\end{cases}$

$$
= \begin{cases}0, & \text { if } k=N_{p} \\ 1-\mathcal{F}^{(1)}\left(E_{t}\right), & \text { if } k=N_{p}-1, \\ \int_{0}^{E_{t}} f^{\left(N_{p}-k-1\right)}(y)\left(1-\mathcal{F}^{(1)}\left(E_{t}-y\right)\right) \mathrm{d} y, & \text { if } 1 \leq k \leq N_{p}-2, \\ \mathcal{F}^{\left(N_{p}-1\right)}\left(E_{t}\right), & \text { if } k=0 .\end{cases}
$$

Using Lemma 2 and Remark 1, we show that the PMF of $K$, for a given $E_{t}>0$, depends on the value of $\varepsilon_{p s}$.

- If $\varepsilon_{p s}=0$,

$$
\mathcal{P}_{K}(k)= \begin{cases}0, & \text { if } k=N_{p}, \\ \frac{\left(\frac{E_{t}}{\zeta \varepsilon_{s}}\right)^{2\left(N_{p}-k-1\right)}\left(1+\frac{1}{2\left(N_{p}-k\right)-1} \frac{E_{t}}{\zeta \varepsilon_{s}}\right)}{\Gamma\left(2\left(N_{p}-k\right)-1\right)} e^{-\frac{E_{t}}{\zeta s_{s}},}, & \text { if } 1 \leq k \leq N_{p}-1, \\ \frac{\gamma\left(2\left(N_{p}-1\right) \frac{E_{t}}{\zeta s_{s}}\right)}{\Gamma\left(2\left(N_{p}-1\right)\right)}, & \text { if } k=0 .\end{cases}
$$

- If $\varepsilon_{p s} \neq 0$,

$$
\mathcal{P}_{K}(k)= \begin{cases}0, & \text { if } k=N_{p}, \\ \frac{\varepsilon_{p} e^{-\frac{E_{t}}{\varepsilon t_{p}}}-\varepsilon_{s} e^{-\frac{E_{t}}{\zeta \varepsilon_{s}}}}{\varepsilon_{p s}}, & \text { if } k=N_{p}-1, \\ \frac{\sqrt{2 \pi}\left(\frac{2 \varepsilon_{p} \varepsilon_{s}}{\varepsilon_{s}}\right)^{N_{p}-k-1}}{\Gamma\left(N_{p}-k-1\right) \varepsilon_{p s}}\left(\varepsilon_{p} e^{-\frac{E_{t}}{\zeta \varepsilon_{p}}} D_{k+1}\left(\frac{\varepsilon_{p s}}{\varepsilon_{p} \varepsilon_{s}} \frac{E_{t}}{2 \zeta}, 1\right)\right. & \text { if } 1 \leq k \leq N_{p}-2, \\ -\varepsilon_{s} e^{-\frac{E_{t}}{\zeta \varepsilon_{s}}} D_{k+1}\left(\frac{\varepsilon_{p s}}{\left.\left.\varepsilon_{p} \varepsilon_{s} \frac{E_{t}}{2 \zeta},-1\right)\right),}\right. & \\ \frac{\gamma\left(N_{p}-1, \frac{E_{t}}{\zeta \varepsilon_{p}}\right)}{\Gamma\left(N_{p}-1\right)}-\left(\frac{E_{t}}{\zeta \varepsilon_{p}}\right)^{N_{p}-1} e^{-\frac{E_{t}}{\zeta \varepsilon_{s}}} & \text { if } k=0, \\ \times \sum_{j=0}^{N_{p}-2} \frac{\left(\frac{E_{t}}{\zeta \varepsilon_{s}}\right)_{1}^{j} F_{1}\left(N_{p}-1 ; N_{p}+j ; \frac{\varepsilon_{p s}}{\xi \varepsilon_{s} \varepsilon_{p}} E_{t}\right)}{\Gamma\left(N_{p}+j\right)}, & \end{cases}
$$

with $D_{k}(x, a)=\int_{0}^{x} u^{N_{p}-k-\frac{1}{2}} e^{-a u} I_{N_{p}-k-\frac{1}{2}}(u) \mathrm{d} u$. After some mathematical manipulations, we show that

$$
\begin{aligned}
& D_{k+1}\left(\frac{\varepsilon_{p s}}{\varepsilon_{p} \varepsilon_{s}} \frac{E_{t}}{2 \zeta}, \pm 1\right)=\frac{\left(\frac{\varepsilon_{p s}}{\varepsilon_{p} \varepsilon_{s}} \frac{E_{t}}{2 \zeta}\right)^{N_{p}-k-\frac{3}{2}} e^{\mp \frac{\varepsilon_{p s}}{\varepsilon_{p} \varepsilon_{s}} \frac{E_{t}}{2 \zeta}}}{2\left(N_{p}-k-1\right)} \\
& \times\left[\left(2\left(N_{p}-k-\frac{1}{2}\right) \pm \frac{\varepsilon_{p s}}{\varepsilon_{p} \varepsilon_{s}} \frac{E_{t}}{2 \zeta}\right) I_{N_{p}-k-\frac{1}{2}}\left(\frac{\varepsilon_{p s}}{\varepsilon_{p} \varepsilon_{s}} \frac{E_{t}}{2 \zeta}\right)\right. \\
& \left.+\frac{\varepsilon_{p s}}{\varepsilon_{p} \varepsilon_{s}} \frac{E_{t}}{2 \zeta} I_{N_{p}-k+\frac{1}{2}}\left(\frac{\varepsilon_{p s}}{\varepsilon_{p} \varepsilon_{s}} \frac{E_{t}}{2 \zeta}\right)\right] .
\end{aligned}
$$

Consequently, we deduce that the PMF of $K$ is given by (18), and hence the end of the proof. 
APPENDIX D

Proof of Theorem 2

For a given $1 \leq K \leq N_{p}$, the conditional CDF of $\gamma_{I D}(K)$ is expressed as

$$
\mathcal{F}_{\gamma_{I D}(K)}\left(\xi_{p}\right)=\int_{0}^{\infty} \mathcal{F}_{\gamma_{p p}}\left(\xi_{p}(u+1)\right) f_{\gamma_{s p}}(u) \mathrm{d} u
$$

Given that the PDF of $g_{p p}$ is $f_{g_{p p}}(g)=\frac{1}{\Gamma(K)} \frac{g^{K-1}}{\lambda_{p p}^{K}} e^{-\frac{g}{\lambda_{p p}}}$, and the PDF of $g_{s p}$ is independent of $g_{p p}$ as shown in [29] and is given by $f_{g_{s p}}(w)=\frac{1}{\lambda_{s p}} e^{-\frac{w}{\lambda_{s p}}}$, for $g \geq 0$ and $w \geq 0$, we can write

$$
\begin{aligned}
f_{\gamma_{s p}}(u) & =\frac{1}{\overline{\gamma_{p_{2}}}} f_{g_{s p}}\left(\frac{u}{\overline{\gamma_{p_{2}}}}\right)=\frac{1}{\beta_{2}} e^{-\frac{u}{\beta_{2}}}, \\
\mathcal{F}_{\gamma_{p p}}\left(\xi_{p}(u+1)\right) & =\frac{1}{\Gamma(K)} \gamma\left(K, \frac{\xi_{p}(u+1)}{\beta_{1}}\right) .
\end{aligned}
$$

Subsequently, we obtain

$$
\mathcal{F}_{\gamma_{I D}(K)}\left(\xi_{p}\right)=\frac{\beta_{1}}{\Gamma(K) \beta_{2}} \frac{e^{\frac{1}{\beta_{2}}}}{\xi_{p}} \int_{\frac{\xi_{p}}{\beta_{1}}}^{\infty} \gamma(K, t) e^{-\frac{\beta_{1}}{\beta_{2}} \frac{t}{\xi_{p}}} \mathrm{~d} t .
$$

Then, we substitute the definition of the lower incomplete Gamma function as in $[28,(8.352 .1)]$, permute the double integrals, and we compute the inner integral using [28, (3.381.1)] and $[28,(3.381 .3)]$ to show that, for $a>0, v>0$, and $K \geq 1$, we have

$$
\int_{a}^{\infty} \gamma(K, t) e^{-v t} \mathrm{~d} t=\frac{e^{-v a} \gamma(K, a)+(1+v)^{-K} \Gamma(K,(1+v) a)}{v} .
$$

Applying this equality to (109), we deduce that the conditional CDF of $\gamma_{I D}(K)$ is given by (30).

\section{Appendix E}

Derivation of THE EXPRESSION OF $\mathcal{I}_{2}\left(\xi_{s}\right)$ IN $\mathcal{F}_{\gamma_{s}}\left(\xi_{s}\right)$

Given the expression of $f_{\gamma_{s d}}(x)$ in (86), we can write the integral $\mathcal{I}_{2}\left(\xi_{s}\right)$ as

$$
\begin{aligned}
\mathcal{I}_{2}\left(\xi_{s}\right) & =N_{s} p_{0}\left(\xi_{s}\right) e^{-\frac{\xi_{s}}{\alpha_{1} p_{0}\left(\xi_{s}\right)}}+\frac{N_{s}}{\Gamma\left(N_{p}\right) \alpha_{1}} \sum_{j=1}^{N_{s}-1} \sum_{i=0}^{N_{p}(j+1)-1}\left(\begin{array}{c}
N_{s}-1 \\
j
\end{array}\right) \\
& \times\left(\begin{array}{c}
N_{p}(j+1)-1 \\
i
\end{array}\right)(-1)^{j+i} \int_{\xi_{s}}^{\infty} e^{-\frac{x}{\alpha_{2} \xi_{s}}}\left(\frac{x}{\alpha_{1}}\right)^{i} \Gamma\left(N_{p}-i, \frac{x}{\alpha_{1}}\right) \mathrm{d}
\end{aligned}
$$

Then, we substitute the upper incomplete Gamma function by its sum representation in $[28,(8.352 .2)]$ for $i<N_{p}$, and by its sum representation in $[28,(8.352 .5)]$ for $i>N_{p}$ and $j=1, N_{s}-$ 1. Following that, we use the integral presentation of the upper incomplete Gamma function in [28, (3.381.3)]. Then, we use the integral presentation of $\Gamma\left(0, \frac{x}{\alpha_{1}}\right)$ from $[28,(3.381 .3)]$, we permute the double integrals, and we solve the inner integral using the sum presentation of the upper incomplete Gamma function as in $[28,(8.352 .2)]$ to show that

$$
\begin{aligned}
& \mathcal{I}_{2}\left(\xi_{s}\right)=N_{s} p_{0}\left(\xi_{s}\right) e^{-\frac{\xi_{s}}{\alpha_{1} p_{0}\left(\xi_{s}\right)}}+\frac{N_{s}}{\Gamma\left(N_{p}\right)} \sum_{j=1}^{N_{s}-1}\left(\begin{array}{c}
N_{s}-1 \\
j
\end{array}\right) \\
& \times\left[\sum_{i=0}^{N_{p}-1}\left(\begin{array}{c}
N_{p}(j+1)-1 \\
i
\end{array}\right)(-1)^{j+i} \Gamma\left(N_{p}-i\right) \sum_{l=i+1}^{N_{p}} \frac{\left(p_{0}\left(\xi_{s}\right)\right)^{l} \Gamma\left(l, \frac{\xi_{s}}{\alpha_{1} p_{0}\left(\xi_{s}\right.}\right)}{\Gamma(l-i)}\right)
\end{aligned}
$$

$$
\begin{aligned}
& +\sum_{i=N_{p}}^{N_{p}(j+1)-1}\left(\begin{array}{c}
N_{p}(j+1)-1 \\
i
\end{array}\right) \frac{(-1)^{j+N_{p}}}{\Gamma\left(i-N_{p}+1\right)}\left(\frac{\xi_{s} \alpha_{2}}{\alpha_{1}}\right)^{i+1} \\
& \times\left(\Gamma\left(i+1, \frac{1}{\alpha_{2}}\right) \Gamma\left(0, \frac{\xi_{s}}{\alpha_{1}}\right)-\Gamma(i+1) \sum_{m=0}^{i} \frac{\left(\frac{\alpha_{1} p_{0}\left(\xi_{s}\right)}{\xi_{s} \alpha_{2}}\right)^{m} \Gamma\left(m, \frac{\xi_{s}}{\alpha_{1} p_{0}\left(\xi_{s}\right)}\right)}{\Gamma(m+1)}\right) \\
& -\sum_{i=N_{p}+1}^{N_{p}(j+1)-1}\left(\begin{array}{c}
N_{p}(j+1)-1 \\
i
\end{array}\right) \frac{(-1)^{j+N_{p}}}{\Gamma\left(i-N_{p}+1\right)} \\
& \left.\times \sum_{l=N_{p}+1}^{i+1}(-1)^{i-l} \Gamma(i-l+1)\left(p_{0}\left(\xi_{s}\right)\right)^{l} \Gamma\left(l, \frac{\xi_{s}}{\alpha_{1} p_{0}\left(\xi_{s}\right)}\right)\right] .
\end{aligned}
$$

\section{REFERENCES}

[1] L. Varshney, "Transporting information and energy simultaneously," in IEEE International Symposium on Information Theory (ISIT'2008), Jul. 2008, pp. 1612-1616.

[2] P. Grover and A. Sahai, "Shannon meets Tesla: Wireless information and power transfer," in IEEE International Symposium on Information Theory Proceedings (ISIT'2010), Jun. 2010, pp. 2363-2367.

[3] R. Zhang and C. K. Ho, "MIMO broadcasting for simultaneous wireless information and power transfer," IEEE Transactions on Wireless Communications, vol. 12, no. 5, pp. 1989-2001, May 2013.

[4] I. Krikidis, S. Sasaki, S. Timotheou, and Z. Ding, "A low complexity antenna switching for joint wireless information and energy transfer in MIMO relay channels," IEEE Transactions on Communications, vol. 62, no. 5, pp. 1577-1587, May 2014.

[5] C. Shen, W.-C. Li, and T.-H. Chang, "Simultaneous information and energy transfer: A two-user MISO interference channel case," in IEEE Global Communications Conference (GLOBECOM'2012), Dec. 2012, pp. 3862-3867.

[6] G. Zhu, C. Zhong, H. A. Suraweera, G. K. Karagiannidis, Z. Zhang, and T. A. Tsiftsis, "Wireless information and power transfer in relay systems with multiple antennas and interference," IEEE Transactions on Communications, vol. 63, no. 4, pp. 1400-1418, Apr. 2015.

[7] Z. Ding, C. Zhong, D. W. K. Ng, M. Peng, H. A. Suraweera, R. Schober, and H. V. Poor, "Application of smart antenna technologies in simultaneous wireless information and power transfer," IEEE Communications Magazine, vol. 53, no. 4, pp. 86-93, Apr. 2015.

[8] F. Benkhelifa, A. S. Salem, and M. S. Alouini, "Rate maximization in MIMO Decode-and-Forward communications with an EH relay and possibly imperfect CSI," IEEE Transactions on Communications, vol. 64, no. 11, pp. 4534-4549, Nov. 2016.

[9] F. Benkhelifa and M. S. Alouini, "Precoding design of MIMO Amplifyand-Forward communication system with an energy harvesting relay and possibly imperfect CSI," IEEE Access, vol. 5, pp. 578-594, 2017.

[10] J. Mitola and J. Maguire, G.Q., "Cognitive radio: m," IEEE Personal Communications.

[11] A. Ghasemi and E. Sousa, "Fundamental limits of spectrum-sharing in fading environments," IEEE Transactions on Wireless Communications, vol. 6, no. 2, pp. 649-658, Feb. 2007.

[12] K. Tourki, F. Khan, K. Qaraqe, H.-C. Yang, and M.-S. Alouini, "Exact performance analysis of MIMO cognitive radio systems using transmit antenna selection," IEEE Journal on Selected Areas in Communications, vol. 32, no. 3, pp. 425-438, Mar. 2014.

[13] $\mathrm{D} . \mathrm{Li}$, "Performance analysis of MRC diversity for cognitive radio systems," IEEE Transactions on Vehicular Technology, vol. 61, no. 2, pp. 849-853, Feb. 2012.

[14] R. Duan, M. Elmusrati, R. Jantti, and R. Virrankoski, "Capacity for spectrum sharing cognitive radios with MRC diversity at the secondary receiver under asymmetric fading," in IEEE Global Telecommunications Conference (GLOBECOM'2010), Dec. 2010, pp. 1-5.

[15] M. F. Hanif, P. J. Smith, D. P. Taylor, and P. A. Martin, "MIMO cognitive radios with antenna selection," IEEE Transactions on Wireless Communications, vol. 10, no. 11, pp. 3688-3699, Nov. 2011.

[16] R. Sarvendranath and N. B. Mehta, "Antenna selection in interferenceconstrained underlay cognitive radios: SEP-optimal rule and performance benchmarking," IEEE Transactions on Communications, vol. 61, no. 2, pp. 496-506, Feb. 2013.

S. Lee, R. Zhang, and K. Huang, "Opportunistic wireless energy harvesting in cognitive radio networks," IEEE Transactions on Wireless Communications, vol. 12, no. 9, pp. 4788-4799, Sept. 2013. 
[18] F. Zhu, F. Gao, and M. Yao, "A new cognitive radio strategy for SWIPT system," in International Workshop onHigh Mobility Wireless Communications (HMWC'2014), Nov. 2014, pp. 73-77.

[19] Y. Liu, S. A. Mousavifar, Y. Deng, C. Leung, and M. Elkashlan, "Wireless energy harvesting in a cognitive relay network," IEEE Transactions on Wireless Communications, vol. 15, no. 4, pp. 2498-2508, Apr. 2016.

[20] S. S. Kalamkar and A. Banerjee, "Interference-assisted wireless energy harvesting in cognitive relay network with multiple primary transceivers," in IEEE Global Communications Conference (GLOBECOM'2015), Dec. 2015, pp. 1-6.

[21] G. Im and J. H. Lee, "Outage probability of underlay cognitive radio networks with SWIPT-enabled relay," in IEEE 82nd Vehicular Technology Conference (VTC-Fall'2015), Sept. 2015, pp. 1-5.

[22] A. E. Shafie, N. Al-Dhahir, and R. Hamila, "Cooperative access schemes for efficient SWIPT transmissions in cognitive radio networks," in IEEE Globecom Workshops (Globecom'2015), Dec. 2015, pp. 1-6.

[23] L. Sibomana, H. J. Zepernick, and H. Tran, "Wireless information and power transfer in an underlay cognitive radio network," in 8th International Conference on Signal Processing and Communication Systems (ICSPCS'2014), Dec. 2014, pp. 1-7.

[24] C. Xu, M. Zheng, W. Liang, H. Yu, and Y. C. Liang, "Outage performance of underlay multihop cognitive relay networks with energy harvesting," IEEE Communications Letters, vol. 20, no. 6, pp. 1148 1151, Jun. 2016.

[25] Z. Yang, Z. Ding, P. Fan, and G. K. Karagiannidis, "Outage performance of cognitive relay networks with wireless information and power transfer," IEEE Transactions on Vehicular Technology, vol. 65, no. 5, pp. 3828-3833, May 2016.

[26] K. Tourki and M. Hasna, "Proactive spectrum sharing incentive for physical layer security enhancement," in IEEE Global Communications Conference, Exhibition \& Industry Forum (Globecom'2015), San Diego, CA, USA, Dec. 2015.

[27] K. Tourki and M. O. Hasna, "Proactive spectrum sharing incentive for physical layer security enhancement using outdated CSI," IEEE Transactions on Wireless Communications, vol. 15, no. 9, pp. 62736283, Sept. 2016

[28] I. S. Gradshteyn and I. M. Ryzhik, Table of Integrals, Series, and Products, 5th ed. San Diego, CA: Academic, 1994.

[29] M. Kang and M.-S. Alouini, "A comparative study on the performance of MIMO MRC systems with and without co-channel interference," in IEEE International Conference on Communications (ICC'2003), vol. 3, May 2003, pp. 2154-2158 vol.3. 